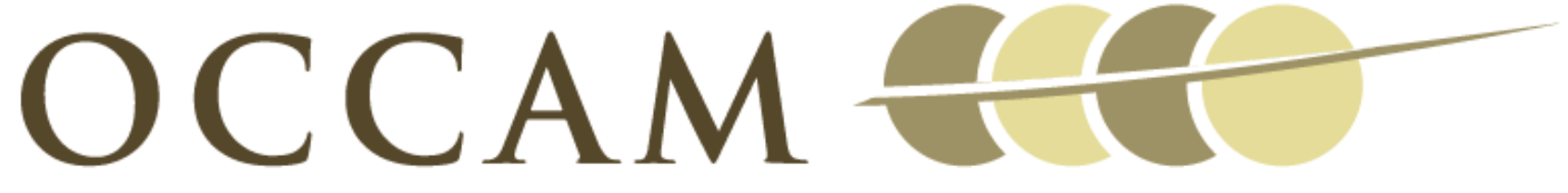

OXFORD CENTRE FOR COLLABORATIVE APPLIED MATHEMATICS

Report Number 09/30

An hp-Local Discontinuous Galerkin method for Parabolic Integro-Differential Equations

by

Amiya K. Pani and Sangita Yadav

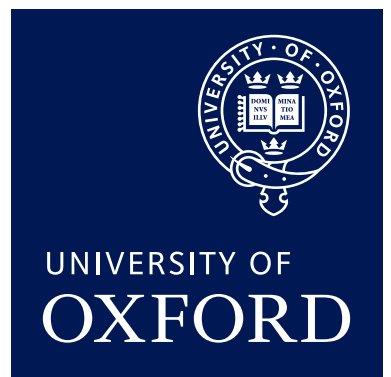

Oxford Centre for Collaborative Applied Mathematics Mathematical Institute 24 - 29 St Giles'

Oxford

OX1 3LB

England 



\title{
An $h p$-Local Discontinuous Galerkin method for Parabolic Integro-Differential Equations
}

\author{
Amiya K. Pani and Sangita Yadav
}

\begin{abstract}
In this article, a priori error analysis is discussed for an hp-local discontinuous Galerkin (LDG) approximation to a parabolic integro-differential equation. It is shown that the $L^{2}$-norm of the gradient and the $L^{2}$-norm of the potential are optimal in the discretizing parameter $h$ and suboptimal in the degree of polynomial $p$. Due to the presence of the integral term, an introduction of an expanded mixed type Ritz-Volterra projection helps to achieve optimal estimates. Further, it is observed that a negative norm estimate of the gradient plays a crucial role in our convergence analysis. As in the elliptic case, similar results on order of convergence are established for the semidiscrete method after suitably modifying the numerical fluxes. The optimality of these theoretical results is tested in a series of numerical experiments on two dimensional domains.
\end{abstract}

Key words. Parabolic integro-differential equation, local discontinuous Galerkin method, semidiscrete optimal error estimate.

\section{Introduction}

In this article, we discuss locally discontinuous Galerkin (LDG) method for the following parabolic integro-differential equation:

$$
\begin{aligned}
u_{t}-\nabla \cdot\left(a(x) \nabla u+\int_{0}^{t} b(x, t, s) \nabla u(s) d s\right) & =f(x, t) & & \text { in } \Omega \times(0 T], \\
u(x, t) & =0 & & \text { on } \partial \Omega \times(0 T], \\
u_{\mid t=0} & =u_{0} & & \text { in } \Omega,
\end{aligned}
$$

where $u_{t}=\frac{\partial u}{\partial t}$. We assume that $\Omega$ is a bounded domain in $\mathbb{R}^{2}$ with smooth boundary $\partial \Omega$, $a(x)$ and $b(x, t, s)$ are smooth bounded functions in $\Omega$, and there exist positive constants $\alpha_{0}, M$ such that $0<\alpha_{0} \leq a \leq M,|b(x, t, s)| \leq M$. Parabolic integro-differential equations (PIDE) of the above type arise naturally in many applications, such as, heat conduction in materials with memory, nonlocal reactive flows in porous media and Non-Fickian flow of fluid in porous media, see [2], [6], [10]-[14] and [18].

In the past, the semidiscrete Galerkin approximation to parabolic integro-differential equations with smooth and nonsmooth initial data was studied and discussed by Thomée and Zhang [18], Cannon and Lin [2], Lin et. al. [10], Pani and Peterson [12] and Pani and Sinha [13]. In literature, Pani and Fairweather [11], Ewing et. al. [6,7] and references therein have proposed and analyzed mixed finite element methods for the problem (1.1)-(1.3). 
In recent years, there has been a renewed interest in Discontinuous Galerkin (DG) methods for numerical approximations of partial differential equations. This is due to their flexibility in local mesh adaptivity and in handling nonuniform degrees of approximation for solutions whose smoothness exhibit variation over the computational domain. DG methods have an advantage that they are element-wise conservative and easy to implement compared to other numerical methods such as finite element methods, mixed finite element methods and finite volume element methods. One such method in the family of DG methods is locally discontinuous Galerkin (LDG) method which allows arbitrary meshes with hanging nodes, elements of various shapes and piecewise polynomials of different degrees. It is originally initiated for a system of first order hyperbolic problems [5], but Cockburn and Shu [5] have introduced the LDG method as an extension to general convection-diffusion problems. Subsequently, Castillo et. al. in [4] have discussed $h p$-version of the LDG method for a convection-diffusion problem. The application of LDG method to elliptic problems is carried by Cockburn et. al. [3], Perugia and Schötzau [15] and Gudi et. al. [8]. In [3], the authors have discussed stability and order of convergence of the LDG method applied to the Poisson equation while Perugia and Schötzau in [15] have analyzed $h p$-estimates for linear elliptic problem, and Gudi et. al. in [8] have established $h p$-estimates for nonlinear elliptic problems. Shu and his collaborators have extensively analyzed the LDG method for higher order partial differential equations. For more detail, see $[5,9,19,20]$ and references therein.

In this paper, an $h p$-LDG method is applied to the problem (1.1)-(1.3) and a priori error analysis is discussed. It is proved that if polynomials of degree at least $p$ are used in all the elements, the rate of convergence of the LDG method in the $L^{2}$-norm of $u$ and its velocity $\mathbf{q}=\nabla u$ are of order $p+1 / 2$ and $p$, respectively, when the stabilization parameter $C_{11}$ is taken to be of order one. When the stabilization parameter $C_{11}$ is taken to be of order $h^{-1}$, the order of convergence of $u$ is shown to be $p+1$, as expected. Due to the presence of the integral term, an introduction of expanded mixed type Ritz-Volterra projection helps to achieve optimal estimates. Moreover, it is observed that a negative norm estimate of the gradient plays a crucial role in our error analysis. Our numerical experiments for the LDG method have all been performed with different degree of polynomials and yield optimal order of convergence of $p+1$ and $p$ for $u$ and $\mathbf{q}=\nabla u$, respectively. Throughout this paper, $C$ denotes a generic positive constant which does not depend on the discretizing parameter $h$ and degree of polynomial $p$.

The rest of the article is organized as follows. In section 2, preliminaries and basic results are noted. Section 3 is devoted to the LDG method. In section 4 , we have discussed the estimates for intermediate Ritz-Volterra projection. Section 5 deals with a priori error estimates. In section 6, numerical experiments are conducted to illustrate the theoretical results. We conclude section 7 with some remarks.

\section{Preliminaries}

Let $\mathcal{T}_{h}=\left\{K_{i}: 1 \leq i \leq N_{h}\right\}$ be a shape regular finite element subdivision of $\Omega$, where $K_{i}$ is either a triangle or rectangle. Let $h_{i}$ be the diameter of $K_{i}$ and $h=\max \left\{h_{i}: 1 \leq i \leq N_{h}\right\}$. We denote the set of interior edges of $\mathcal{T}_{h}$ by $\Gamma_{I}=\left\{e_{i j}: e_{i j}=\partial K_{i} \cap \partial K_{j},\left|e_{i j}\right|>0\right\}$ and boundary edges by $\Gamma_{\partial}=\left\{e_{i \partial}: e_{i \partial}=\partial K_{i} \cap \partial \Omega,\left|e_{i \partial}\right|>0\right\}$, where $\left|e_{k}\right|$ denotes the one dimensional Euclidean measure. Let $\Gamma=\Gamma_{I} \cup \Gamma_{\partial}$. Note that our definition of $e_{k}$ also includes hanging nodes along each side of the finite elements. On this subdivision $\mathcal{T}_{h}$, we define the following 
broken Sobolev Spaces

$$
V=\left\{v \in L^{2}(\Omega):\left.v\right|_{K_{i}} \in H^{1}\left(K_{i}\right), \forall K_{i} \in \mathcal{T}_{h}\right\}
$$

and

$$
\mathbf{W}=\left\{\mathbf{w} \in \mathbf{L}^{2}(\Omega):\left.\mathbf{w}\right|_{K_{i}} \in \mathbf{H}^{1}\left(K_{i}\right), \forall K_{i} \in \mathcal{T}_{h}\right\},
$$

where $H^{1}\left(K_{i}\right)$ is the standard Sobolev space of order one defined on $K_{i}, \mathbf{L}^{2}(\Omega)=\left(L^{2}(\Omega)\right)^{2}$ and $\mathbf{H}^{1}(\Omega)=\left(H^{1}(\Omega)\right)^{2}$. The associated broken norm and seminorm on $\mathrm{V}$ are defined, respectively, as

$$
\|v\|_{H^{1}\left(\Omega, \mathcal{T}_{h}\right)}=\left(\sum_{i=1}^{N_{h}}\|v\|_{H^{1}\left(K_{i}\right)}^{2}\right)^{\frac{1}{2}} \text { and }|v|_{H^{1}\left(\Omega, \mathcal{T}_{h}\right)}=\left(\sum_{i=1}^{N_{h}}|v|_{H^{1}\left(K_{i}\right)}^{2}\right)^{\frac{1}{2}} .
$$

We denote the $L^{2}$ inner product by $(\cdot, \cdot)$ and norm by $\|\cdot\|$.

Let $e_{k} \in \Gamma_{I}$, that is $e_{k}=\partial K_{i} \cup \partial K_{j}$ for some $i$ and $j$. Let $\boldsymbol{\nu}_{i}$ and $\boldsymbol{\nu}_{j}$ be the outward normals to the boundary $\partial K_{i}$ and $\partial K_{j}$, respectively. On $e_{k}$, we now define the jump and average of $v \in V$ as

$$
\llbracket v \rrbracket=\left.v\right|_{K_{i}} \boldsymbol{\nu}_{i}+\left.v\right|_{K_{j}} \boldsymbol{\nu}_{j}, \quad\{\{v\}\}=\frac{\left.v\right|_{K_{i}}+\left.v\right|_{K_{j}}}{2},
$$

respectively, and for $\mathbf{w} \in \mathbf{W}$, the jump and average define as

$$
\llbracket \mathbf{w} \rrbracket=\left.\mathbf{w}\right|_{K_{i}} \cdot \boldsymbol{\nu}_{i}+\left.\mathbf{w}\right|_{K_{j}} \cdot \boldsymbol{\nu}_{j}, \quad\{\mathbf{w}\}=\frac{\left.\mathbf{w}\right|_{K_{i}}+\left.\mathbf{w}\right|_{K_{j}}}{2} .
$$

In case, $e_{k} \in \partial \Omega$, that is, there exists $K_{i}$ such that $e_{k}=\partial K_{i} \cap \partial \Omega$, then set the jump and average for $v$ as

$$
\llbracket v \rrbracket=\left.v\right|_{K_{i} \cap \partial \Omega} \boldsymbol{\nu}, \quad\left\{\{v\}=\left.v\right|_{K_{i} \cap \partial \Omega},\right.
$$

respectively, and for $\mathbf{w} \in \mathbf{W}$, the jump and average are defined respectively by

$$
\llbracket \mathbf{w} \rrbracket=\left.\mathbf{w}\right|_{K_{i} \cap \partial \Omega} \cdot \nu, \quad\{\mathbf{w}\}=\left.\mathbf{w}\right|_{K_{i} \cap \partial \Omega},
$$

where $\boldsymbol{\nu}$ is the outward normal to the boundary $\partial \Omega$. Let $P_{p_{i}}\left(K_{i}\right)$ be the space of polynomials of degree less than or equal to $p_{i}$ on each triangle $K_{i} \in \mathcal{T}_{h}$ and $Q_{p_{i}}\left(K_{i}\right)$ be the space of polynomials of degree less than or equal to $p_{i}$ in each variable which are defined on the rectangles $K_{i} \in \mathcal{T}_{h}$. The discontinuous finite element spaces are considered as

$$
V_{h}=\left\{v_{h} \in L^{2}(\Omega):\left.v_{h}\right|_{K_{i}} \in Z_{p_{i}}\left(K_{i}\right)\right\},
$$

and

$$
\mathbf{W}_{h}=\left\{\mathbf{w}_{h} \in\left(\mathbf{L}^{2}(\Omega)\right): \mathbf{w}_{h} \in \mathbf{Z}_{p_{i}}\left(K_{i}\right)\right\},
$$

where $\mathbf{Z}_{p_{i}}\left(K_{i}\right)=\left(Z_{p_{i}}\left(K_{i}\right)\right)^{2}, p_{i} \geq 1$ and $Z_{p_{i}}\left(K_{i}\right)$ is either $P_{p_{i}}\left(K_{i}\right)$ or $Q_{p_{i}}\left(K_{i}\right)$. For any $e_{k} \in \Gamma_{I}$, there are two elements $K_{i}$ and $K_{j}$ such that $e_{k}=\partial K_{i} \cap \partial K_{j}$. We associate $h_{k}$ and $p_{k}$ to $e_{k}$ where $h_{k}$ is either $h_{i}$ or $h_{j}$ and $p_{k}$ is either $p_{i}$ or $p_{j}$. For $e_{k} \in \Gamma_{\partial}$, since there is one element $K_{i}$ such that $e_{k}=\partial K_{i} \cap \partial \Omega$, we write $h_{k}=h_{i}$ and $p_{k}=p_{i}$. We also denote

$$
h=\max _{1 \leq i \leq N_{h}} h_{i} \text { and } p=\min _{1 \leq i \leq N_{h}} p_{i}
$$

Assumption (P). The finite element subdivision $\mathcal{T}_{h}$ satisfies the bounded local variation in the sense that for any neighbor elements $\mathrm{K}$ and $K^{\prime} \in \mathcal{T}_{h}$, there exits a constant $\rho$ such that

$$
\frac{h_{K}}{h_{K^{\prime}}} \leq \rho
$$

Below, we state a Lemma on the approximation properties of the finite element spaces whose proof can be found in [1]. 
Lemma 2.1. For $\phi \in\left(H^{r}\left(K_{i}\right)\right)^{d}, d=1,2$, there exists a positive constant $C_{A}$ (depending on $r$ but independent of $\phi, p_{i}$ and $h_{i}$ ) and a sequence $\phi_{p}^{h} \in Z_{p_{i}}\left(K_{i}\right)^{d}, p_{i} \geq 1$, such that

- for any $0 \leq l \leq s$,

$$
\left\|\phi-\phi_{p}^{h}\right\|_{\left(H^{l}\left(K_{i}\right)\right)^{d}} \leq C_{A} \frac{h_{i}^{\min \left\{r, p_{i}+1\right\}-l}}{p_{i}^{r-l}}\|\phi\|_{\left(H^{r}\left(K_{i}\right)\right)^{d}},
$$

- for $l \leq r-\frac{1}{2}$,

$$
\left\|\phi-\phi_{p}^{h}\right\|_{\left(H^{l}\left(e_{k}\right)\right)^{d}} \leq C_{A} \frac{h_{i}^{\min \left\{r, p_{i}+1\right\}-l-\frac{1}{2}}}{p_{i}^{r-l-\frac{1}{2}}}\|\phi\|_{\left(H^{r}\left(K_{i}\right)\right)^{d}}
$$

- for $0 \leq l \leq r-1+\frac{2}{m}$,

$$
\left\|\phi-\phi_{p}^{h}\right\|_{\left(W_{m}^{l}\left(e_{k}\right)\right)^{d}} \leq C_{A} \frac{h_{i}^{\min \left\{r, p_{i}+1\right\}-l-1+\frac{2}{m}}}{p_{i}^{r-l-1+\frac{2}{m}}}\|\phi\|_{\left(H^{r}\left(K_{i}\right)\right)^{d}} .
$$

We now denote $I_{h} \phi=\phi_{p}^{h}$ on each $K_{i}$.

Trace inequality. We shall use the following trace inequality on the finite element spaces. For a proof, we refer to [16].

Lemma 2.2. (Trace inequality) Let $v_{h} \in\left(Z_{p_{i}}\left(K_{i}\right)\right)^{d}, d=1,2$. Then there exist a constant $C_{T}>0$ such

$$
\left\|\nabla^{l} v_{h}\right\|_{\left(L^{2}\left(e_{k}\right)\right)^{d}} \leq C_{T} p_{i} h_{i}^{-\frac{1}{2}}\left\|\nabla^{l} v_{h}\right\|_{\left(L^{2}\left(K_{i}\right)\right)^{d}}, \quad l=0,1 .
$$

Lemma 2.3. ( $L^{2}$-projection $\left.\Pi\right)$. Let $\boldsymbol{\psi} \in\left(H^{r+1}\left(K_{i}\right)\right)^{2}$ and $\boldsymbol{\psi}_{h}=\Pi \boldsymbol{\psi} \in\left(Z_{p_{i}}\left(K_{i}\right)\right)^{2}$ be the $L^{2}$-projection of $\boldsymbol{\psi}$ onto $\left(Z_{p_{i}}\left(K_{i}\right)\right)^{2}$. Then the following approximation property holds:

$$
\left\|\boldsymbol{\psi}-\boldsymbol{\psi}_{h}\right\|_{\left(L^{2}\left(K_{i}\right)\right)^{2}}+\frac{h_{i}^{\frac{1}{2}}}{p_{i}}\left\|\boldsymbol{\psi}-\boldsymbol{\psi}_{h}\right\|_{\left(L^{2}\left(\partial K_{i}\right)\right)^{2}} \leq C \frac{h_{i}^{\min \left(r, p_{i}\right)+1}}{p_{i}^{r+1}}\|\boldsymbol{\psi}\|_{\left(H^{r+1}\left(K_{i}\right)\right)^{2}}
$$

\section{Local Discontinuous Galerkin Method}

The LDG method was originally initiated for a system of first order hyperbolic problems [5]. To define the method for parabolic integro-differential equations (1.1)-(1.3), we first introduce auxiliary variables

$$
\mathbf{q}=\nabla u, \quad \boldsymbol{\sigma}=a \mathbf{q}+\int_{0}^{t} b(t, s) \mathbf{q} d s,
$$

and then rewrite (1.1) as a system of equations:

$$
\begin{aligned}
\mathbf{q} & =\nabla u & & \text { in } \Omega, \\
\boldsymbol{\sigma} & =a \mathbf{q}+\int_{0}^{t} b(t, s) \mathbf{q} d s & & \text { in } \Omega, \\
u_{t}-\nabla \cdot \boldsymbol{\sigma} & =f & & \text { in } \Omega .
\end{aligned}
$$


We now multiply (3.1) by $\mathbf{w} \in \mathbf{W},(3.2)$ by $\boldsymbol{\tau} \in \mathbf{W}$ and (3.3) by $v \in V$ and then we integrate over the element $K \in \mathcal{T}_{h}$ and use integration by parts formula to obtain

$$
\begin{aligned}
\int_{K} \mathbf{q} \cdot \mathbf{w} d x+\int_{K} u \nabla \cdot \mathbf{w} d x-\int_{\partial K} u \mathbf{w} \cdot \boldsymbol{\nu}_{K} d S & =0 \\
\int_{K} a \mathbf{q} \cdot \boldsymbol{\tau} d x-\int_{K} \boldsymbol{\sigma} \cdot \boldsymbol{\tau} d x+\int_{0}^{t} \int_{K} b(t, s) \mathbf{q}(s) \cdot \boldsymbol{\tau} d x d s & =0 \\
\int_{K} u_{t} v d x+\int_{K} \boldsymbol{\sigma} \cdot \nabla v d x-\int_{\partial K} \boldsymbol{\sigma} \cdot \boldsymbol{\nu}_{K} v d s & =\int_{K} f v d x .
\end{aligned}
$$

Note that there may be difficulty in defining $u$ and $\boldsymbol{\sigma}$ on $\partial \Omega$. Therefore, the system (3.4)(3.6) is useful in defining the LDG method. For all $K \in \mathcal{T}_{h}$, we seek approximate solution $\left(u_{h}, \mathbf{q}_{\mathbf{h}}, \boldsymbol{\sigma}_{h}\right) \in Z_{p}(K) \times \mathbf{Z}_{p}(K) \times \mathbf{Z}_{p}(K)$ satisfying the following system of equations for all $\left(v_{h}, \mathbf{w}_{h}, \boldsymbol{\tau}_{h}\right) \in Z_{p}(K) \times \mathbf{Z}_{p}(K) \times \mathbf{Z}_{p}(K):$

$$
\begin{array}{r}
\int_{K} \mathbf{q}_{h} \cdot \mathbf{w}_{h}+\int_{K} u_{h} \nabla \cdot \mathbf{w}_{h}-\int_{\partial K} \hat{u} \mathbf{w}_{h} \cdot \boldsymbol{\nu}_{K} d s=0, \\
\int_{K} a \mathbf{q}_{h} \cdot \boldsymbol{\tau}_{h} d x-\int_{K} \boldsymbol{\sigma}_{h} \cdot \boldsymbol{\tau}_{h} d x+\int_{0}^{t} \int_{K} b(t, s) \mathbf{q}_{h}(s) \cdot \boldsymbol{\tau}_{h} d x d s=0, \\
\int_{K} u_{h t} v_{h} d x+\int_{K} \boldsymbol{\sigma}_{h} \cdot \nabla v_{h} d x-\int_{\partial K} \hat{\boldsymbol{\sigma}} \cdot \boldsymbol{\nu}_{K} v_{h} d s=\int_{K} f v_{h} d x,
\end{array}
$$

where the numerical fluxes $\hat{u}$ and $\hat{\boldsymbol{\sigma}}$ have to be suitably chosen in order to ensure the stability of the method and also to improve the order of convergence. As in the case of linear elliptic problem, we use the following choice of numerical fluxes. For $e_{k} \in \Gamma$, the numerical fluxes are defined on $e_{k}$ as:

$$
\begin{aligned}
\hat{u}\left(u_{h}, \boldsymbol{\sigma}_{h}\right) & =\left\{\left\{u_{h}\right\}+C_{12} \cdot \llbracket u_{h} \rrbracket-C_{22} \llbracket \boldsymbol{\sigma}_{h} \rrbracket,\right. \\
\hat{\boldsymbol{\sigma}}\left(u_{h}, \boldsymbol{\sigma}_{h}\right) & =\left\{\left\{\boldsymbol{\sigma}_{h}\right\}-C_{11} \llbracket u_{h} \rrbracket-C_{12} \llbracket \boldsymbol{\sigma}_{h} \rrbracket .\right.
\end{aligned}
$$

The numerical fluxes are conservative since they are single valued on $e_{k} \in \Gamma_{I}$, that is, on $e_{k} \in \Gamma_{I}$,

$$
\llbracket \hat{u} \rrbracket=0, \quad \llbracket \hat{\boldsymbol{\sigma}} \rrbracket=0,
$$

and consistent since for smooth $\mathrm{u}$ and $\boldsymbol{\sigma}$, the following conditions hold:

$$
\begin{aligned}
\hat{u}(u, \boldsymbol{\sigma}) & =u, \\
\hat{\boldsymbol{\sigma}}(u, \boldsymbol{\sigma}) & =\boldsymbol{\sigma} .
\end{aligned}
$$

This completes the definition of our DG method. The LDG method is obtained when $C_{22}=0$, that is when the numerical flux $\hat{u}_{h}$ does not depend on $\boldsymbol{\sigma}_{h}$.

In order to obtain a LDG method, we sum (3.7)-(3.9) over all elements $K \in \mathcal{T}_{h}$. Then using the conservative property (3.12) and the definition of numerical fluxes, we obtain the following system of equations for all $\left(v_{h}, \mathbf{w}_{h}, \boldsymbol{\tau}_{h}\right) \in V_{h} \times \mathbf{W}_{h} \times \mathbf{W}_{h}$ :

$$
\begin{gathered}
\int_{\Omega} \mathbf{q}_{h} \cdot \mathbf{w}_{h}+\sum_{i=1}^{N_{h}} \int_{K_{i}} u_{h} \nabla \cdot \mathbf{w}_{h}-\int_{\Gamma_{I}}\left(\left\{u_{h} \rrbracket\right\}+C_{12} \cdot \llbracket u_{h} \rrbracket-C_{22} \llbracket \boldsymbol{\sigma}_{h} \rrbracket\right) \llbracket \mathbf{w}_{h} \rrbracket d s=0, \\
\int_{\Omega} a \mathbf{q}_{h} \cdot \boldsymbol{\tau}_{h} d x-\int_{\Omega} \boldsymbol{\sigma}_{h} \cdot \boldsymbol{\tau}_{h} d x+\int_{0}^{t} \int_{\Omega} b(t, s) \mathbf{q}_{h}(s) \cdot \boldsymbol{\tau}_{h} d x d s=0, \\
\left.\int_{\Omega} u_{h t} v_{h} d x+\sum_{i=1}^{N_{h}} \int_{K_{i}} \boldsymbol{\sigma}_{h} \cdot \nabla v_{h} d x-\int_{\Gamma}\left(\left\{\boldsymbol{\sigma}_{h}\right\}\right\}-C_{11} \llbracket u_{h} \rrbracket-C_{12} \llbracket \boldsymbol{\sigma}_{h} \rrbracket\right) \llbracket v_{h} \rrbracket d s=\int_{K} f v_{h} d x .
\end{gathered}
$$


For $(\phi, \mathbf{p}),(v, \mathbf{w}) \in V \times \mathbf{W}$, define the bilinear functional: $A: \mathbf{W} \times \mathbf{W} \rightarrow \mathbb{R}$ as

$$
A(\mathbf{p}, \mathbf{w})=\int_{\Omega} \mathbf{p} \cdot \mathbf{w} d x
$$

$A_{1}: V \times \mathbf{W} \rightarrow \mathbb{R}$ as

$$
\begin{aligned}
A_{1}(v, \mathbf{p}) & \left.=\sum_{i=1}^{N_{h}} \int_{K_{i}} \mathbf{p} \cdot \nabla v d x-\int_{\Gamma}(\{\mathbf{p}\}\}-C_{12} \llbracket \mathbf{p} \rrbracket\right) \llbracket v \rrbracket d s, \\
& \left.=-\sum_{i=1}^{N_{h}} \int_{K_{i}} v \nabla \cdot \mathbf{p}+\int_{\Gamma_{I}}(\{v\}]+C_{12} \cdot \llbracket v \rrbracket\right) \llbracket \mathbf{p} \rrbracket d s,
\end{aligned}
$$

$J_{1}: \mathbf{W} \times \mathbf{W} \rightarrow \mathbb{R}$ as

$$
J_{1}(\mathbf{p}, \mathbf{w})=\int_{\Gamma_{I}} C_{22} \llbracket \mathbf{p} \rrbracket \llbracket \mathbf{w} \rrbracket d S
$$

$A_{2}: \mathbf{W} \times \mathbf{W} \rightarrow \mathbb{R}$ as

$$
A_{2}(\mathbf{p}, \mathbf{w})=\int_{\Omega} a \mathbf{p} \cdot \mathbf{w} d x
$$

$B: \mathbf{W} \times \mathbf{W} \rightarrow \mathbb{R}$ as

$$
B(t, s, \mathbf{p}, \mathbf{w})=\int_{\Omega} b(t, s) \mathbf{p} \cdot \mathbf{w} d x
$$

and $J: V \times V \rightarrow \mathbb{R}$ as

$$
J(\phi, v)=\int_{\Gamma_{I}} C_{11} \llbracket \phi \rrbracket \llbracket v \rrbracket d S .
$$

We also define the linear functional $L: V \rightarrow \mathbb{R}$ as

$$
L(v)=\int_{\Omega} f v d x .
$$

Using the above definitions, we rewrite the LDG method for the problem (3.15)-(3.17) in compact form as : find $\left(u_{h}, \mathbf{q}_{h}, \boldsymbol{\sigma}_{h}\right):[0, T] \rightarrow V_{h} \times \mathbf{W}_{h} \times \mathbf{W}_{h}$ such that for all $\left(v_{h}, \boldsymbol{\tau}_{h}, \mathbf{w}_{h}\right) \in$ $V_{h} \times \mathbf{W}_{h} \times \mathbf{W}_{h}$ the following hold:

$$
\begin{array}{r}
A\left(\mathbf{q}_{h}, \mathbf{w}_{h}\right)-A_{1}\left(u_{h}, \mathbf{w}_{h}\right)+J_{1}\left(\boldsymbol{\sigma}_{h}, \mathbf{w}_{h}\right)=0, \\
A_{2}\left(\mathbf{q}_{h}, \boldsymbol{\tau}_{h}\right)-A\left(\boldsymbol{\sigma}_{h}, \boldsymbol{\tau}_{h}\right)+\int_{0}^{t} B\left(t, s, \mathbf{q}_{h}(s), \boldsymbol{\tau}_{h}\right) d s=0, \\
\left(u_{h t}, v_{h}\right)+A_{1}\left(\boldsymbol{\sigma}_{h}, v_{h}\right)+J\left(u_{h}, v_{h}\right)=L\left(v_{h}\right) .
\end{array}
$$

We need to introduce the set $\left\langle K, K^{\prime}\right\rangle$ defined as

$$
\left\langle K, K^{\prime}\right\rangle=\left\{\begin{array}{lr}
\emptyset & \text { if meas }\left(\partial K \cap \partial K^{\prime}\right)=0, \\
\text { interior of } \partial K \cap \partial K^{\prime} & \text { otherwise. }
\end{array}\right.
$$

We assume that the stabilization coefficients $C_{11}$ and $C_{22}$ defining the numerical fluxes in (3.10) and (3.11) are defined as

$$
C_{11}(x)= \begin{cases}\zeta \min \left\{h_{K^{+}}^{\alpha} p_{K^{+}}^{\gamma}, h_{K^{-}}^{\alpha} p_{K^{-}}^{\gamma}\right\} & \text { if } \boldsymbol{x} \in\left\langle K^{+}, K^{-}\right\rangle \\ \zeta h_{K^{+}}^{\alpha} p_{K^{+}}^{\gamma} & \text { if } \boldsymbol{x} \in \partial K^{+} \cap \partial \Omega\end{cases}
$$


and

$$
C_{22}(x)= \begin{cases}\kappa \min _{i}\left\{h_{K^{+}}^{\beta} p_{K^{+}}^{\delta}, h_{K^{-}}^{\beta} p_{K^{-}}^{\delta}\right\} & \text { if } \boldsymbol{x} \in\left\langle K^{+}, K^{-}\right\rangle, \\ \kappa h_{K^{+}}^{\beta} p_{K^{+}}^{\delta^{+}} & \text {if } \boldsymbol{x} \in \partial K^{+} \cap \partial \Omega,\end{cases}
$$

with $\zeta>0, \kappa \geq 0,-1 \leq \alpha \leq 0 \leq \beta \leq 1,-2 \leq \gamma \leq 0 \leq \delta \leq 2$ independent of mesh size and $\left|C_{12}\right|$ of order one. Our main result will be written in terms of the parameters $\mu^{*}$ and $\mu_{*}$ defined by

$$
\mu^{*}=\max \{-\alpha, \hat{\beta}\}, \quad \mu_{*}=\min \{-\alpha, \hat{\beta}\},
$$

where $\hat{\beta}=1$ if $\kappa=0$ and $\hat{\beta}=\beta$ otherwise. For each internal or boundary edge, we set

$$
\chi(\boldsymbol{x}):=\left\{\begin{array}{lr}
\min \left\{\frac{h_{K}}{p_{K}^{2}}, \frac{h_{K^{\prime}}}{p_{K^{\prime}}^{2}}\right\} \text { for } \boldsymbol{x} \in\left\langle K, K^{\prime}\right\rangle, \frac{h_{K}}{p_{K}^{2}} \text { for } \boldsymbol{x} \in \partial K \cap \partial \Omega & \text { if } C_{22}(\boldsymbol{x})=0, \\
C_{22}(\boldsymbol{x}) & \text { otherwise }
\end{array}\right.
$$

Since the numerical fluxes $\hat{u}$ and $\hat{\boldsymbol{\sigma}}$ are consistent, we easily obtain the following system of equations, for all $\left(v_{h}, \boldsymbol{\tau}_{h}, \mathbf{w}_{h}\right) \in V_{h} \times \mathbf{W}_{h} \times \mathbf{W}_{h}$,

$$
\begin{array}{r}
A\left(\mathbf{q}-\mathbf{q}_{h}, \mathbf{w}_{h}\right)-A_{1}\left(u-u_{h}, \mathbf{w}_{h}\right)+J_{1}\left(\boldsymbol{\sigma}-\boldsymbol{\sigma}_{h}, \mathbf{w}_{h}\right)=0, \\
A_{2}\left(\mathbf{q}-\mathbf{q}_{h}, \boldsymbol{\tau}_{h}\right)-A\left(\boldsymbol{\sigma}-\boldsymbol{\sigma}_{h}, \boldsymbol{\tau}_{h}\right)+\int_{0}^{t} B\left(t, s,\left(\mathbf{q}-\mathbf{q}_{h}\right)(s), \boldsymbol{\tau}_{h}\right) d s=0, \\
\left(u_{t}-u_{h t}, v_{h}\right)+A_{1}\left(\boldsymbol{\sigma}-\boldsymbol{\sigma}_{h}, v_{h}\right)+J\left(u-u_{h}, v_{h}\right)=0 .
\end{array}
$$

Below, we only state the main theorem of this article whose proof can be found in section 5 .

Theorem 3.1. Let $(u, \mathbf{q}, \boldsymbol{\sigma})$ be the solution of (3.1)-(3.3) and $\left(u_{h}, \mathbf{q}_{h}, \boldsymbol{\sigma}_{h}\right) \in V_{h} \times \mathbf{W}_{h} \times \mathbf{W}_{h}$ be the solution of (3.18)-(3.20). If $u_{h}(0)=I_{h} u_{0}$ and $q_{h}(0)=I_{h} \nabla u_{0}$, then the following estimates hold:

$$
\left\|u-u_{h}\right\| \leq C \frac{h^{P+D}}{p^{Q+Z}}\left(\|u\|_{H^{r+2}}+\int_{0}^{t}\left\{\|u(s)\|_{H^{r+2}}+\left\|u_{s}(s)\right\|_{H^{r+2}}\right\} d s\right),
$$

and

$$
\left\|\mathbf{q}-\mathbf{q}_{h}\right\|+\left\|\boldsymbol{\sigma}-\boldsymbol{\sigma}_{h}\right\| \leq C \frac{h^{P}}{p^{Q}}\left(\|u\|_{H^{r+2}}+\int_{0}^{t}\left\{\|u(s)\|_{H^{r+2}}+\left\|u_{s}(s)\right\|_{H^{r+2}}\right\} d s\right),
$$

where $P=\min \left\{r+\frac{1}{2}\left(1+\mu_{*}\right), p+\frac{1}{2}\left(1-\mu^{*}\right)\right\}, D=\frac{1}{2}\left(1+\mu_{*}\right), Q=r+Z$ and $Z=\frac{1}{2} \min \{1, \gamma,-\delta\}$.

Below, we have presented a table which gives the order of convergence in $h$ with different parameters. Note that the order in $h p$ is easy to infer from (3.27)-(3.28).

Table 1: Orders of convergence for $u \in H^{r+2}(\Omega)$ for $r \geq 0$ and $p \geq 0$.

\begin{tabular}{|lc|c|c|}
\hline$C_{22}$ & $C_{11}$ & $\left\|\mathbf{q}-\mathbf{q}_{h}\right\|,\left\|\boldsymbol{\sigma}-\boldsymbol{\sigma}_{h}\right\|$ & $\left\|u-u_{h}\right\|$ \\
\hline $0, O(h)$ & $O(1)$ & $\min \left\{r+\frac{1}{2}, p\right\}$ & $\min \left\{r+\frac{1}{2}, p\right\}+\frac{1}{2}$ \\
$0, O(h)$ & $O(1 / h)$ & $\min \{r+1, p\}$ & $\min \{r+1, p\}+1$ \\
\hline$O(1)$ & $O(1)$ & $\min \{r, p\}+\frac{1}{2}$ & $\min \{r, p\}+1$ \\
$O(1)$ & $O(1 / h)$ & $\min \left\{r+\frac{1}{2}, p\right\}$ & $\min \left\{r+\frac{1}{2}, p\right\}+\frac{1}{2}$ \\
\hline
\end{tabular}




\section{Ritz-Volterra Projection and Related Estimates}

For our subsequent analysis, we define the following extended mixed Ritz-Volterra projection: Find $\left(\tilde{u}_{h}, \tilde{\mathbf{q}}_{h}, \tilde{\boldsymbol{\sigma}}_{h}\right):[0, T] \rightarrow V_{h} \times \mathbf{W}_{h} \times \mathbf{W}_{h}$ satisfying:

$$
\begin{array}{r}
A\left(\mathbf{q}-\tilde{\mathbf{q}}_{h}, \mathbf{w}_{h}\right)-A_{1}\left(u-\tilde{u}_{h}, \mathbf{w}_{h}\right)+J_{1}\left(\boldsymbol{\sigma}-\tilde{\boldsymbol{\sigma}}_{h}, \mathbf{w}_{h}\right)=0 \quad \forall \mathbf{w}_{h} \in \mathbf{W}_{h}, \\
A_{2}\left(\mathbf{q}-\tilde{\mathbf{q}}_{h}, \tau_{h}\right)-A\left(\boldsymbol{\sigma}-\tilde{\boldsymbol{\sigma}}_{h}, \boldsymbol{\tau}_{h}\right)+\int_{0}^{t} B\left(t, s,\left(\mathbf{q}-\tilde{\mathbf{q}}_{h}\right)(s), \boldsymbol{\tau}_{h}\right) d s=0 \quad \forall \boldsymbol{\tau}_{h} \in \mathbf{W}_{h}, \\
A_{1}\left(\boldsymbol{\sigma}-\tilde{\boldsymbol{\sigma}}_{h}, v_{h}\right)+J\left(u-\tilde{u}_{h}, v_{h}\right)=0 \quad \forall v_{h} \in V_{h} .
\end{array}
$$

For given $(u, \mathbf{q}, \boldsymbol{\sigma})$, it is easy to prove existence and uniqueness of the solution $\left(\tilde{u}_{h}, \tilde{q}_{h}, \tilde{\boldsymbol{\sigma}}_{h}\right)$ of (4.1)-(4.3).

Using $I_{h}$ and $\Pi_{h}$ projections, we write

$$
\begin{aligned}
u-\tilde{u}_{h} & =\left(u-I_{h} u\right)-\left(\tilde{u}_{h}-I_{h} u\right)=\theta_{u}-\rho_{u}, \\
\mathbf{q}-\tilde{\mathbf{q}}_{h} & =\left(\mathbf{q}-I_{h} \mathbf{q}\right)-\left(\tilde{\mathbf{q}}_{h}-I_{h} \mathbf{q}\right)=\boldsymbol{\theta}_{\mathbf{q}}-\boldsymbol{\rho}_{\mathbf{q}}, \\
\boldsymbol{\sigma}-\tilde{\boldsymbol{\sigma}}_{h} & =\left(\boldsymbol{\sigma}-\Pi_{h} \boldsymbol{\sigma}\right)-\left(\tilde{\boldsymbol{\sigma}}_{h}-\Pi_{h} \boldsymbol{\sigma}\right)=\boldsymbol{\theta}_{\boldsymbol{\sigma}}-\boldsymbol{\rho}_{\boldsymbol{\sigma}},
\end{aligned}
$$

and hence, from (4.1)-(4.3), we obtain

$$
\begin{aligned}
A\left(\boldsymbol{\rho}_{\mathbf{q}}, \mathbf{w}_{h}\right)-A_{1}\left(\rho_{u}, \mathbf{w}_{h}\right)+J_{1}\left(\boldsymbol{\rho}_{\boldsymbol{\sigma}}, \mathbf{w}_{h}\right) & =A\left(\boldsymbol{\theta}_{\mathbf{q}}, \mathbf{w}_{h}\right)-A_{1}\left(\mathbf{w}_{h}, \theta_{u}\right)+J_{1}\left(\boldsymbol{\theta}_{\boldsymbol{\sigma}}, \mathbf{w}_{h}\right), \\
A_{2}\left(\boldsymbol{\rho}_{\mathbf{q}}, \boldsymbol{\tau}_{h}\right)-A\left(\boldsymbol{\rho}_{\boldsymbol{\sigma}}, \boldsymbol{\tau}_{h}\right)+\int_{0}^{t} B\left(t, s, \boldsymbol{\rho}_{\mathbf{q}}(s), \boldsymbol{\tau}_{h}\right) d s & =A_{2}\left(\boldsymbol{\theta}_{\mathbf{q}}, \boldsymbol{\tau}_{h}\right)+\int_{0}^{t}\left(B\left(t, s, \boldsymbol{\theta}_{\mathbf{q}}(s), \boldsymbol{\tau}_{h}\right) d s,\right. \\
A_{1}\left(\boldsymbol{\rho}_{\boldsymbol{\sigma}}, v_{h}\right)+J\left(\rho_{u}, v_{h}\right) & =A_{1}\left(\boldsymbol{\theta}_{\boldsymbol{\sigma}}, v_{h}\right)+J\left(\theta_{u}, v_{h}\right) .
\end{aligned}
$$

Below, we discuss the estimates of $\left\|\rho_{\mathbf{q}}\right\|$ and $\left\|\rho_{\boldsymbol{\sigma}}\right\|$.

Lemma 4.1. There is a positive constant $C$ independent of $h$ and $p$ such that

$$
\left\|\boldsymbol{\rho}_{\mathbf{q}}\right\|^{2}+\left\|\boldsymbol{\rho}_{\boldsymbol{\sigma}}\right\|^{2}+\int_{\Gamma} C_{11} \llbracket \rho_{u} \rrbracket^{2} d S+\int_{\Gamma} C_{22} \llbracket \boldsymbol{\rho}_{\boldsymbol{\sigma}} \rrbracket^{2} d S \leq C \frac{h^{2 P}}{p^{2 Q}}\left(\|u\|_{H^{r+2}}^{2}+\int_{0}^{t}\|u(s)\|_{H^{r+2}}^{2} d s\right),
$$

where

$$
P=\min \left\{r+\frac{1}{2}\left(1+\mu_{*}\right), p+\frac{1}{2}\left(1-\mu^{*}\right)\right\}, \quad Q=r+\frac{1}{2} \min \{1, \delta,-\gamma\}
$$

Proof. Choose $\boldsymbol{\tau}_{h}=\boldsymbol{\rho}_{\boldsymbol{\sigma}}$ in (4.5) and use Cauchy-Schwarz inequality with $\alpha_{0} \leq a \leq M$ and $|b(t, s)| \leq M$ to find that

$$
\begin{aligned}
\left\|\boldsymbol{\rho}_{\boldsymbol{\sigma}}\right\|^{2} & =A_{2}\left(\boldsymbol{\rho}_{\mathbf{q}}, \boldsymbol{\rho}_{\boldsymbol{\sigma}}\right)+\int_{0}^{t} B\left(t, s, \boldsymbol{\rho}_{\mathbf{q}}(s), \boldsymbol{\rho}_{\boldsymbol{\sigma}}(t) d s-A_{2}\left(\boldsymbol{\theta}_{\mathbf{q}}, \boldsymbol{\rho}_{\boldsymbol{\sigma}}\right)-\int_{0}^{t} B\left(t, s, \boldsymbol{\theta}_{\mathbf{q}}(s), \boldsymbol{\rho}_{\boldsymbol{\sigma}}(t) d s\right.\right. \\
& \leq C(M)\left(\left\|\boldsymbol{\rho}_{\mathbf{q}}\right\|+\left\|\boldsymbol{\theta}_{\mathbf{q}}\right\|+\int_{0}^{t}\left\|\boldsymbol{\rho}_{q}(s)\right\| d s+\int_{0}^{t}\left\|\boldsymbol{\theta}_{\mathbf{q}}(s)\right\| d s\right)\left\|\boldsymbol{\rho}_{\boldsymbol{\sigma}}\right\|,
\end{aligned}
$$

and hence,

$$
\left\|\boldsymbol{\rho}_{\boldsymbol{\sigma}}\right\| \leq C(M)\left(\left\|\boldsymbol{\rho}_{\mathbf{q}}\right\|+\left\|\boldsymbol{\theta}_{\mathbf{q}}\right\|+\int_{0}^{t}\left(\left\|\boldsymbol{\rho}_{\mathbf{q}}(s)\right\|+\left\|\boldsymbol{\theta}_{\mathbf{q}}(s)\right\|\right) d s\right)
$$


Now, choose $\mathbf{w}_{h}=\boldsymbol{\rho}_{\boldsymbol{\sigma}}$ in (4.4), $\boldsymbol{\tau}_{h}=\boldsymbol{\rho}_{\mathbf{q}}$ in (4.5) and $v_{h}=\rho_{u}$ in (4.6) and then sum up to obtain

$$
\begin{aligned}
A_{2}\left(\boldsymbol{\rho}_{\mathbf{q}}, \boldsymbol{\rho}_{\mathbf{q}}\right)+J\left(\rho_{u}, \rho_{u}\right)+J_{1}\left(\boldsymbol{\rho}_{\boldsymbol{\sigma}}, \boldsymbol{\rho}_{\boldsymbol{\sigma}}\right)= & A\left(\boldsymbol{\theta}_{\mathbf{q}}, \boldsymbol{\rho}_{\boldsymbol{\sigma}}\right)-A_{1}\left(\boldsymbol{\rho}_{\boldsymbol{\sigma}}, \theta_{u}\right)+J_{1}\left(\boldsymbol{\theta}_{\boldsymbol{\sigma}}, \boldsymbol{\rho}_{\boldsymbol{\sigma}}\right)+A_{2}\left(\boldsymbol{\theta}_{\mathbf{q}}, \boldsymbol{\rho}_{\mathbf{q}}\right) \\
& +A_{1}\left(\boldsymbol{\theta}_{\boldsymbol{\sigma}}, \rho_{u}\right)+J\left(\theta_{u}, \rho_{u}\right)+\int_{0}^{t} B\left(t, s, \boldsymbol{\theta}_{\mathbf{q}}(s), \boldsymbol{\rho}_{\mathbf{q}}(t)\right) d s \\
& -\int_{0}^{t}\left(B\left(t, s, \boldsymbol{\rho}_{\mathbf{q}}(s), \boldsymbol{\rho}_{\mathbf{q}}(t)\right) d s .\right.
\end{aligned}
$$

A use of Cauchy-Schwarz inequality yields

$$
\left|A\left(\boldsymbol{\theta}_{\mathbf{q}}, \boldsymbol{\rho}_{\boldsymbol{\sigma}}\right)\right|=\left|\sum_{K} \int_{K} \boldsymbol{\theta}_{\mathbf{q}} \cdot \boldsymbol{\rho}_{\boldsymbol{\sigma}} d x\right| \leq \sum_{K}\left\|\boldsymbol{\theta}_{\mathbf{q}}\right\|_{K}\left\|\boldsymbol{\rho}_{\boldsymbol{\sigma}}\right\|_{K} \leq\left\|\boldsymbol{\theta}_{\mathbf{q}}\right\|\left\|\boldsymbol{\rho}_{\boldsymbol{\sigma}}\right\|,
$$

and using (4.8), we arrive at

$$
\left|A\left(\boldsymbol{\theta}_{\mathbf{q}}, \boldsymbol{\rho}_{\boldsymbol{\sigma}}\right)\right| \leq C\left(\left\|\boldsymbol{\theta}_{\mathbf{q}}\right\|^{2}+\int_{0}^{t}\left\|\boldsymbol{\theta}_{\mathbf{q}}\right\|^{2} d s\right)+\varepsilon\left\|\boldsymbol{\rho}_{\mathbf{q}}\right\|^{2}+C \int_{0}^{t}\left\|\boldsymbol{\rho}_{\mathbf{q}}\right\|^{2} d s .
$$

We note from the definition that

$$
A_{1}\left(\boldsymbol{\rho}_{\boldsymbol{\sigma}}, \theta_{u}\right)=\sum_{K \in \mathcal{T}_{h}} \int_{K} \theta_{u} \nabla \cdot \boldsymbol{\rho}_{\boldsymbol{\sigma}} d x-\int_{\Gamma_{I}}\left\{\theta_{u}\right\} \llbracket \llbracket \boldsymbol{\rho}_{\boldsymbol{\sigma}} \rrbracket d S-\int_{\Gamma_{I}} C_{12} \llbracket \theta_{u} \rrbracket \llbracket \boldsymbol{\rho}_{\boldsymbol{\sigma}} \rrbracket d S .
$$

Integration by parts and use of Lemma 2.2 yields

$$
\sum_{K \in \mathcal{T}_{h}} \int_{K} \theta_{u} \nabla \cdot \boldsymbol{\rho}_{\boldsymbol{\sigma}} d x-\int_{\Gamma_{I}}\left\{\theta_{u}\right\} \llbracket \llbracket \boldsymbol{\rho}_{\boldsymbol{\sigma}} \rrbracket d S=-\sum_{K \in \mathcal{T}_{h}} \int_{K} \nabla \theta_{u} \cdot \boldsymbol{\rho}_{\boldsymbol{\sigma}} d x+\int_{\Gamma} \llbracket \theta_{u} \rrbracket\left\{\boldsymbol{\rho}_{\boldsymbol{\sigma}}\right\} d S,
$$

and hence

$$
|B i g| \sum_{K \in \mathcal{T}_{h}} \int_{K} \nabla \theta_{u} \cdot \boldsymbol{\rho}_{\boldsymbol{\sigma}} d x-\int_{\Gamma} \llbracket \theta_{u} \rrbracket\left\{\left\{\boldsymbol{\rho}_{\boldsymbol{\sigma}}\right\}\right\} d S \mid \leq C\left(\left\|\nabla \theta_{u}\right\|^{2}+\int_{\Gamma} \frac{p_{K}^{2}}{h_{K}} \llbracket \theta_{u} \rrbracket^{2}\right)^{\frac{1}{2}}\left\|\boldsymbol{\rho}_{\boldsymbol{\sigma}}\right\| .
$$

For the third term on the right hand side of (4.11), again, an application of Cauchy-Schwarz yields

$$
\left|\int_{\Gamma_{I}} C_{12} \llbracket \boldsymbol{\theta}_{\boldsymbol{u}} \rrbracket \llbracket \boldsymbol{\rho}_{\boldsymbol{\sigma}} \rrbracket d S\right| \leq C\left(\int_{\Gamma} \chi^{-1} \llbracket \theta_{u} \rrbracket^{2} d S\right)^{\frac{1}{2}}\left(\int_{\Gamma} \chi \llbracket \boldsymbol{\rho}_{\boldsymbol{\sigma}} \rrbracket^{2} d S\right)^{\frac{1}{2}}
$$

On combining (4.13) and (4.14), we obtain using (4.8) that

$$
\left|A_{1}\left(\boldsymbol{\rho}_{\boldsymbol{\sigma}}, \theta_{u}\right)\right| \leq\left(\left\|\boldsymbol{\theta}_{\mathbf{q}}\right\|^{2}+\left\|\nabla \theta_{u}\right\|^{2}+\int_{\Gamma}\left(\frac{p_{K}^{2}}{h_{K}}+\chi^{-1}\right) \llbracket \theta_{u} \rrbracket^{2} d S\right)+\varepsilon\left\|\boldsymbol{\rho}_{\mathbf{q}}\right\|^{2}+C \int_{0}^{t}\left\|\boldsymbol{\rho}_{\mathbf{q}}\right\|^{2} d s .
$$

A simple application of Cauchy-Schwarz inequality now implies

$$
\left|J_{1}\left(\boldsymbol{\theta}_{\boldsymbol{\sigma}}, \boldsymbol{\rho}_{\boldsymbol{\sigma}}\right)\right| \leq J_{1}\left(\boldsymbol{\theta}_{\boldsymbol{\sigma}}, \boldsymbol{\theta}_{\boldsymbol{\sigma}}\right)^{\frac{1}{2}} J_{1}\left(\boldsymbol{\rho}_{\boldsymbol{\sigma}}, \boldsymbol{\rho}_{\boldsymbol{\sigma}}\right)^{\frac{1}{2}} .
$$

From the definition of $A_{1}$ and $J$, we write

$$
A_{1}\left(\boldsymbol{\theta}_{\boldsymbol{\sigma}}, \rho_{u}\right)+J\left(\theta_{u}, \rho_{u}\right)=\sum_{K \in \mathcal{T}_{h}} \int_{K} \boldsymbol{\theta}_{\boldsymbol{\sigma}} \cdot \nabla \rho_{u} d x-\int_{\Gamma}\left(\left\{\boldsymbol{\theta}_{\boldsymbol{\sigma}} \rrbracket-C_{12} \llbracket \boldsymbol{\theta}_{\boldsymbol{\sigma}} \rrbracket-C_{11} \llbracket \theta_{u} \rrbracket\right) \llbracket \rho_{u} \rrbracket d S .\right.
$$


Since $\boldsymbol{\Pi}_{\boldsymbol{h}} \boldsymbol{\sigma}$ is $L^{2}$ projection of $\boldsymbol{\sigma}$ onto $\mathbf{W}_{h}$, it follows that

$$
\sum_{K \in \mathcal{T}_{h}} \int_{K} \boldsymbol{\theta}_{\boldsymbol{\sigma}} \cdot \nabla \rho_{u} d x=0
$$

and hence,

$$
\begin{aligned}
& \left|\int_{\Gamma}\left(\left\{\boldsymbol{\theta}_{\boldsymbol{\sigma}}\right\}-C_{11} \llbracket \theta_{u} \rrbracket-C_{12} \llbracket \boldsymbol{\theta}_{\boldsymbol{\sigma}} \rrbracket\right) \llbracket \rho_{u} \rrbracket d S\right| \leq C \sum_{K \in \mathcal{T}_{h}}\left(C_{11}^{-1}\left\|\boldsymbol{\theta}_{\boldsymbol{\sigma}}\right\|_{\partial K}^{2}+C_{11}\left\|\theta_{u}\right\|_{\partial K}^{2}\right) \\
& +\varepsilon J\left(\rho_{u}, \rho_{u}\right) \text {. }
\end{aligned}
$$

An appeal to the Cauchy-Schwarz inequality yields

$$
\left|A_{2}\left(\boldsymbol{\theta}_{\mathbf{q}}, \boldsymbol{\rho}_{\mathbf{q}}\right)\right| \leq C\left\|\boldsymbol{\theta}_{\mathbf{q}}\right\|\left\|\boldsymbol{\rho}_{\mathbf{q}}\right\| \leq C\left\|\boldsymbol{\theta}_{\mathbf{q}}\right\|^{2}+\varepsilon\left\|\boldsymbol{\rho}_{\mathbf{q}}\right\|^{2} .
$$

A simple application of Cauchy-Schwarz inequality yields a bound for the last term of (4.9) as:

$$
\begin{aligned}
\mid \int_{0}^{t} B\left(t, s, \boldsymbol{\theta}_{\mathbf{q}}(s), \boldsymbol{\rho}_{\mathbf{q}}(t)\right) d s- & \int_{0}^{t} B\left(t, s, \boldsymbol{\rho}_{\mathbf{q}}(s), \boldsymbol{\rho}_{\mathbf{q}}(t)\right) d s \mid \leq C\left\|\boldsymbol{\rho}_{\mathbf{q}}\right\| \int_{0}^{t}\left(\left\|\boldsymbol{\theta}_{\mathbf{q}}\right\|+\left\|\boldsymbol{\rho}_{\mathbf{q}}\right\|\right) d s \\
& \leq C \int_{0}^{t}\left\|\boldsymbol{\theta}_{\mathbf{q}}\right\|^{2} d s+\varepsilon\left\|\boldsymbol{\rho}_{\mathbf{q}}\right\|^{2}+C \int_{0}^{t}\left\|\boldsymbol{\rho}_{\mathbf{q}}\right\|^{2} d s .
\end{aligned}
$$

Substitute the estimates (4.10)-(4.19) in (4.9), use $\alpha_{0} \leq a(x) \leq M, \forall x \in \Omega$, Lemma 2.1 and Lemma 2.3. With the help of a suitable choice of $\varepsilon$, we arrive at

$$
\begin{aligned}
\left\|\boldsymbol{\rho}_{\mathbf{q}}\right\|^{2}+J\left(\rho_{u}, \rho_{u}\right)+J_{1}\left(\boldsymbol{\rho}_{\boldsymbol{\sigma}}, \boldsymbol{\rho}_{\boldsymbol{\sigma}}\right) & \leq C\left[\sum _ { K } \left\{\frac{h_{K}^{2 \min \left\{r, p_{K}\right\}+2}}{p_{K}^{2 r+2}}\|\mathbf{q}\|_{r+1, K}^{2}+\left(C_{11}^{-1}+C_{22}\right) \frac{h_{K}^{2 \min \left\{r, p_{K}\right\}+1}}{p_{K}^{2 r}}\|\boldsymbol{\sigma}\|_{r+1, K}^{2}\right.\right. \\
& \left.\left.+\left(\frac{h_{K}^{2 \min \left\{r+1, p_{K}\right\}}}{p_{K}^{2 r+2}}+\left(\frac{p_{K}^{2}}{h_{K}}+\chi^{-1}+C_{11}\right) \frac{h_{K}^{2 \min \left\{r+1, p_{K}\right\}+1}}{p_{K}^{2 r+3}}\right)\|u\|_{r+2, K}^{2}\right\}\right] \\
& +\int_{0}^{t} \sum_{K} \frac{h_{K}^{2 \min \left\{r, p_{K}\right\}+2}}{p_{K}^{2 r+2}}\|\mathbf{q}(s)\|_{r+1, K}^{2} d s+\int_{0}^{t}\left\|\boldsymbol{\rho}_{\mathbf{q}}(\tau)\right\|^{2} d \tau .
\end{aligned}
$$

Hence, we obtain

$$
\left\|\boldsymbol{\rho}_{\mathbf{q}}\right\|^{2}+J\left(\rho_{u}, \rho_{u}\right)+J_{1}\left(\boldsymbol{\rho}_{\boldsymbol{\sigma}}, \boldsymbol{\rho}_{\boldsymbol{\sigma}}\right) \leq C \frac{h^{2 P}}{p^{2 Q}}\left(\|u\|_{H^{r+2}}^{2}+\int_{0}^{t}\|u(s)\|_{H^{r+2}}^{2} d s\right)+\int_{0}^{t}\left\|\boldsymbol{\rho}_{\mathbf{q}}(\tau)\right\|^{2} d \tau .
$$

An application of Gronwall's Lemma yields the desired estimate for $\left\|\rho_{\mathbf{q}}\right\|$. On substituting the estimate of $\left\|\rho_{\mathbf{q}}\right\|$ in (4.8), we obtain the desired result and this completes the proof.

Theorem 4.1. There exists a positive constant $C$ independent of $h$ and $p$ such that

$$
\left\|\boldsymbol{\sigma}-\tilde{\boldsymbol{\sigma}}_{h}\right\|^{2}+\left\|\mathbf{q}-\tilde{\mathbf{q}}_{h}\right\|^{2}+\int_{\Gamma} C_{11} \llbracket u-\tilde{u}_{h} \rrbracket^{2} d S \leq C \frac{h^{2 P}}{p^{2 Q}}\left(\|u\|_{H^{r+2}}^{2}+\int_{0}^{t}\|u\|_{H^{r+2}}^{2} d s\right) .
$$

Further,

$$
\left\|\mathbf{q}-\tilde{\mathbf{q}}_{h}\right\|_{\left(\mathbf{H}^{1}(\Omega)\right)^{*}} \leq C\left\|u-\tilde{u}_{h}\right\|+C \frac{h^{(P+D)}}{p^{(Q+Z)}}\left(\|u\|_{H^{r+2}}+\int_{0}^{t}\|u\|_{H^{r+2}} d s\right),
$$

where

$$
\|\mathbf{v}\|_{\left(\mathbf{H}^{1}(\Omega)\right)^{*}}=\sup _{\mathbf{w} \in \mathbf{H}^{1}(\Omega), \mathbf{w} \neq 0} \frac{(\mathbf{v}, \mathbf{w})}{\|\mathbf{w}\|_{\mathbf{H}^{1}(\Omega)}} .
$$


Proof. A use of triangle inequality with Lemma 4.1, Lemma 2.1 and Lemma 2.3, easily yields (4.20). For (4.21), we first set $\eta_{u}=u-\tilde{u}_{h}, \boldsymbol{\eta}_{\mathbf{q}}=\mathbf{q}-\tilde{\mathbf{q}}_{h}$ and $\boldsymbol{\eta}_{\boldsymbol{\sigma}}=\boldsymbol{\sigma}-\tilde{\boldsymbol{\sigma}}_{h}$. For any $\mathbf{w} \in \mathbf{H}^{1}(\Omega)$ and $\mathbf{w}_{h} \in \mathbf{w}_{h}$, we obtain, using (4.1)

$$
\begin{aligned}
\left(\boldsymbol{\eta}_{\mathbf{q}}, \mathbf{w}\right) & =\left(\boldsymbol{\eta}_{\mathbf{q}}, \mathbf{w}-\mathbf{w}_{h}\right)+\left(\boldsymbol{\eta}_{\mathbf{q}}, \mathbf{w}_{h}\right) \\
& =\left(\boldsymbol{\eta}_{\mathbf{q}}, \mathbf{w}-\mathbf{w}_{h}\right)-\int_{\Omega} \eta_{u} \nabla \cdot \mathbf{w}_{h} d x+\int_{\Gamma}\left(\left\{\left\{\eta_{u}\right\}\right)+C_{12} \llbracket \eta_{u} \rrbracket\right) \llbracket \mathbf{w}_{h} \rrbracket d S .
\end{aligned}
$$

Since $\llbracket \mathbf{w} \rrbracket=0 \quad \forall \mathbf{w} \in \mathbf{H}^{1}(\Omega)$, we arrive at

$$
\begin{aligned}
\left(\boldsymbol{\eta}_{\mathbf{q}}, \mathbf{w}\right) & =\left(\boldsymbol{\eta}_{\mathbf{q}}, \mathbf{w}-\mathbf{w}_{h}\right)-\sum_{i=1}^{N_{h}} \int_{K_{i}} \eta_{u} \nabla \cdot \mathbf{w} d x+\sum_{i=1}^{N_{h}} \int_{K_{i}} \eta_{u} \nabla \cdot\left(\mathbf{w}-\mathbf{w}_{h}\right) d x \\
& -\int_{\Gamma_{I}}\left\{\left\{\eta_{u} \rrbracket \llbracket \mathbf{w}-\mathbf{w}_{h} \rrbracket d S-\int_{\Gamma_{I}} C_{12} \llbracket \eta_{u} \rrbracket \llbracket \mathbf{w}-\mathbf{w}_{h} \rrbracket d S .\right.\right.
\end{aligned}
$$

Choose $\mathbf{w}_{h}=\Pi_{h} \mathbf{w}$ to obtain

$$
\left|\left(\boldsymbol{\eta}_{\mathbf{q}}, \mathbf{w}-\Pi_{h} \mathbf{w}\right)\right| \leq C \sum_{i=1}^{N_{h}}\left\|\eta_{\mathbf{q}}\right\|_{L^{2}\left(K_{i}\right)}\left\|\mathbf{w}-\Pi_{h} \mathbf{w}\right\|_{\mathbf{L}^{2}\left(K_{i}\right)} .
$$

A use of Lemma 2.3 now yields

$$
\left|\left(\boldsymbol{\eta}_{\mathbf{q}}, \mathbf{w}-\Pi_{h} \mathbf{w}\right)\right| \leq C\left(\sum_{i=1}^{N_{h}} \frac{h_{i}^{2}}{p_{i}^{2}}\left\|\eta_{\mathbf{q}}\right\|_{L^{2}\left(K_{i}\right)}^{2}\right)^{\frac{1}{2}}\|\mathbf{w}\|_{\mathbf{H}^{1}(\Omega)},
$$

and

$$
\left|\sum_{K} \int_{K} \eta_{u} \nabla \cdot \mathbf{w} d x\right| \leq\left\|\eta_{u}\right\|\|\mathbf{w}\|_{\mathbf{H}^{1}(\Omega)} .
$$

Apply integration by parts to the third and fourth terms on the right hand side of (4.22) and obtain

$$
\begin{aligned}
\sum_{K} \int_{K} \eta_{u} \nabla \cdot\left(\mathbf{w}-\Pi_{h} \mathbf{w}\right) d x-\int_{\Gamma_{I}}\left\{\left\{\eta_{u} \rrbracket \llbracket \mathbf{w}-\Pi_{h} \mathbf{w} \rrbracket d S\right.\right. & =-\sum_{i=1}^{N_{h}} \int_{K_{i}} \nabla \eta_{u} \cdot\left(\mathbf{w}-\Pi_{h} \mathbf{w}\right) d x \\
& +\int_{\Gamma} \llbracket \eta_{u} \rrbracket \llbracket \mathbf{w}-\Pi_{h} \mathbf{w} \rrbracket d S .
\end{aligned}
$$

Noting that $\eta_{u}=\theta_{u}-\rho_{u}$ and $\Pi_{h}$ is $L^{2}$ projection, we use Cauchy-Swartz inequality to find that

$$
\begin{aligned}
\mid \sum_{K} \int_{K} \eta_{u} \nabla \cdot\left(\mathbf{w}-\Pi_{h} \mathbf{w}\right) d x- & \int_{\Gamma_{I}}\left\{\left\{\eta_{u}\right\} \llbracket \llbracket \mathbf{w}-\Pi_{h} \mathbf{w} \rrbracket d S \mid \leq\left(\sum_{K} \frac{h_{K}^{2}}{p_{K}^{2}}\left\|\nabla \theta_{u}\right\|_{K}^{2}\right.\right. \\
& \left.+\sum_{e_{k} \in \Gamma} C_{11}^{-1} h_{k} \int_{e_{k}} C_{11} \llbracket \eta_{u} \rrbracket^{2} d S\right)^{\frac{1}{2}}\|\mathbf{w}\|_{\mathbf{H}^{1}(\Omega)} .
\end{aligned}
$$

For the last term on the right hand side of (4.22), we use Cauchy-Schwarz inequality and property of $\Pi_{h}$ to obtain

$$
\begin{aligned}
\left|\int_{\Gamma} C_{12} \llbracket \eta_{u} \rrbracket \llbracket \mathbf{w}-\Pi_{h} \mathbf{w} \rrbracket d S\right| & \leq C \sum_{e_{k} \in \Gamma}\left(\int_{e_{k}} C_{11} \llbracket \eta_{u} \rrbracket^{2} d S\right)^{\frac{1}{2}}\left(\int_{e_{k}} C_{11}^{-1} \llbracket \mathbf{w}-\Pi_{h} \mathbf{w} \rrbracket^{2} d S\right)^{\frac{1}{2}} \\
& \leq C\left(\sum_{e_{k} \in \Gamma} C_{11}^{-1} h_{k} \int_{e_{k}} C_{11} \llbracket \eta_{u} \rrbracket^{2} d S\right)^{\frac{1}{2}}\|\mathbf{w}\|_{\mathbf{H}^{1}(\Omega)} .
\end{aligned}
$$


On combining the estimates (4.23)-(4.26) and using the Lemma 4.1, it now follows that $\left|\left(\boldsymbol{\eta}_{\mathbf{q}}, \mathbf{w}\right)\right| \leq C\left\{\left[\max \left\{\frac{h_{K}^{2}}{p_{K}^{2}}, C_{11}^{-1} h_{K}, C_{22} h_{K}\right\} \frac{h^{2 P}}{p^{2 Q}}\left(\|u\|_{H^{r+2}}^{2}+\int_{0}^{t}\|u\|_{H^{r+2}}^{2} d s\right)\right]^{1 / 2}+\left\|\eta_{u}\right\|\right\}\|\mathbf{w}\|_{\mathbf{H}^{1}(\Omega)}$, and hence, for $\mathbf{w} \neq 0 \in \mathbf{H}^{1}(\Omega)$

$$
\frac{\left|\left(\boldsymbol{\eta}_{\mathbf{q}}, \mathbf{w}\right)\right|}{\|\mathbf{w}\|_{\mathbf{H}^{1}(\Omega)}} \leq C\left[\frac{h^{2(P+D)}}{p^{2(Q+Z)}}\left(\|u\|_{H^{r+2}}^{2}+\int_{0}^{t}\|u\|_{H^{r+2}}^{2} d s\right)\right]^{\frac{1}{2}}+\left\|\eta_{u}\right\| .
$$

Now on taking supremum over all $\mathbf{w} \neq 0 \in \mathbf{H}^{1}(\Omega)$, we obtain the desired estimate. This completes the proof.

After differentiating (4.4)-(4.6), we obtain

$$
\begin{aligned}
& A\left(\boldsymbol{\rho}_{\mathbf{q}_{t}}, \mathbf{w}_{h}\right)-A_{1}\left(\rho_{u t}, \mathbf{w}_{h}\right)+J_{1}\left(\boldsymbol{\rho}_{\boldsymbol{\sigma} t}, \mathbf{w}_{h}\right)=A\left(\boldsymbol{\theta}_{\mathbf{q}_{t}}, \mathbf{w}_{h}\right)-A_{1}\left(\theta_{u t}, \mathbf{w}_{h}\right)+J_{1}\left(\boldsymbol{\theta}_{\boldsymbol{\sigma} t}, \mathbf{w}_{h}\right), \\
& A_{2}\left(\boldsymbol{\rho}_{\mathbf{q} t}, \boldsymbol{\tau}_{h}\right)-A\left(\boldsymbol{\rho}_{\boldsymbol{\sigma} t}, \boldsymbol{\tau}_{h}\right)+B\left(t, t, \boldsymbol{\rho}_{\mathbf{q}}(t), \boldsymbol{\tau}_{h}\right)+\int_{0}^{t} B_{t}\left(t, s, \boldsymbol{\rho}_{\mathbf{q}}(s), \boldsymbol{\tau}_{h}\right) d s \\
& =A_{2}\left(\boldsymbol{\theta}_{\mathbf{q}_{t}}, \boldsymbol{\tau}_{h}\right)+B\left(t, t, \boldsymbol{\theta}_{\mathbf{q}}(t), \boldsymbol{\tau}_{h}\right)+\int_{0}^{t}\left(B_{t}\left(t, s, \boldsymbol{\theta}_{\mathbf{q}}(s), \boldsymbol{\tau}_{h}\right) d s,\right. \\
& A_{1}\left(\boldsymbol{\rho}_{\boldsymbol{\sigma} t}, v_{h}\right)+J\left(\rho_{u t}, v_{h}\right)=A_{1}\left(\boldsymbol{\theta}_{\boldsymbol{\sigma} t}, v_{h}\right)+J\left(\theta_{u t}, v_{h}\right) .
\end{aligned}
$$

Remark 4.1. We put $\mathbf{w}_{h}=\boldsymbol{\rho}_{\boldsymbol{\sigma} t}, \boldsymbol{\tau}_{h}=\boldsymbol{\rho}_{\mathbf{q}_{t}}, v_{h}=\rho_{u t}$ in (4.27)-(4.29) and after proceeding in a similar way as for finding (4.7), we obtain

$$
\begin{aligned}
\left\|\boldsymbol{\rho}_{\mathbf{q}_{t}}\right\|^{2}+\left\|\boldsymbol{\rho}_{\boldsymbol{\sigma} t}\right\|^{2}+\int_{\Gamma} C_{11} \llbracket \rho_{u t} \rrbracket^{2} d S+\int_{\Gamma} C_{22} \llbracket \boldsymbol{\rho}_{\boldsymbol{\sigma} t} \rrbracket^{2} d S & \leq C \frac{h^{2 P}}{p^{2 Q}}\left[\|u\|_{H^{r+2}}^{2}+\left\|u_{t}\right\|_{H^{r+2}}^{2}\right. \\
& \left.+\int_{0}^{t}\|u(s)\|_{H^{r+2}}^{2} d s\right] .
\end{aligned}
$$

An application of triangle inequality yields

$$
\begin{aligned}
\left\|\boldsymbol{\eta}_{\mathbf{q}_{t}}\right\|^{2}+\left\|\boldsymbol{\eta}_{\boldsymbol{\sigma} t}\right\|^{2}+\int_{\Gamma} C_{11} \llbracket \eta_{u t} \rrbracket^{2} d S+\int_{\Gamma} C_{22} \llbracket \boldsymbol{\eta}_{\boldsymbol{\sigma} t} \rrbracket^{2} d S & \leq C \frac{h^{2 P}}{p^{2 Q}}\left[\|u\|_{H^{r+2}}^{2}+\left\|u_{t}\right\|_{H^{r+2}}^{2}\right. \\
& \left.+\int_{0}^{t}\|u(s)\|_{H^{r+2}}^{2} d s\right] .
\end{aligned}
$$

Theorem 4.2. Let $(u, \mathbf{q}, \boldsymbol{\sigma})$ be the solution of (3.1)-(3.3) and let $\left(\tilde{u}_{h}, \tilde{\mathbf{q}}_{h}, \tilde{\boldsymbol{\sigma}}_{h}\right)$ be the solution of (4.1)-(4.3). Then there exist a positive constant $C$ independent of $h$ and $p$ such that

$$
\left\|u-\tilde{u}_{h}\right\| \leq C \frac{h^{(P+D)}}{p^{(Q+Z)}}\left(\|u\|_{H^{r+2}}+\int_{0}^{t}\|u\|_{H^{r+2}} d s\right) .
$$

and

$$
\left\|u_{t}-\tilde{u}_{h t}\right\| \leq C \frac{h^{(P+D)}}{p^{(Q+Z)}}\left(\|u\|_{H^{r+2}}+\left\|u_{t}\right\|_{H^{r+2}}+\int_{0}^{t}\|u\|_{H^{r+2}} d s\right) .
$$

Proof. For $L^{2}$-estimate (4.32), we appeal to the duality argument. Consider the following auxiliary problem:

$$
\begin{aligned}
-\nabla \cdot(a \nabla \phi) & =\eta_{u} \text { in } \Omega, \\
\phi & =0 \text { on } \partial \Omega,
\end{aligned}
$$


which satisfies the elliptic regularity

$$
\|\phi\|_{H^{2}(\Omega)} \leq C\left\|\eta_{u}\right\|
$$

In order to write the mixed weak formulation, let $\mathbf{p}=\nabla \phi$ and $\boldsymbol{\psi}=-a \mathbf{p}$. Then, we obtain

$$
\begin{array}{cl}
\mathbf{p}=\nabla \phi & \text { in } \Omega, \\
-\boldsymbol{\psi}=a \mathbf{p} & \text { in } \Omega, \\
\nabla \cdot \boldsymbol{\psi}=\eta_{u} & \text { in } \Omega .
\end{array}
$$

We multiply (4.37) by $\eta_{u}$, (4.36) by $\boldsymbol{\eta}_{\mathbf{q}}$ and (4.35) by $\boldsymbol{\eta}_{\sigma}$, then integrate to find that

$$
\begin{aligned}
\left\|\eta_{u}\right\|^{2}= & \int_{\Omega} \eta_{u} \nabla \cdot \boldsymbol{\psi} d x+\int_{\Omega} a(x) \mathbf{p} \cdot \boldsymbol{\eta}_{\mathbf{q}} d x+\int_{\Omega} \boldsymbol{\psi} \cdot \boldsymbol{\eta}_{\mathbf{q}} d x \\
& -\int_{\Omega} \mathbf{p} \cdot \boldsymbol{\eta}_{\boldsymbol{\sigma}} d x+\int_{\Omega} \nabla \phi \cdot \boldsymbol{\eta}_{\boldsymbol{\sigma}} d x .
\end{aligned}
$$

As $\llbracket \phi \rrbracket=0, \llbracket \psi \rrbracket=0$ on $e_{k} \in \Gamma_{I}$ and $\phi=0$ on $\partial \Omega$, we now obtain

$$
\left\|\eta_{u}\right\|^{2}=A\left(\boldsymbol{\eta}_{\mathbf{q}}, \boldsymbol{\psi}\right)-A_{1}\left(\eta_{u}, \boldsymbol{\psi}\right)+J_{1}\left(\boldsymbol{\eta}_{\boldsymbol{\sigma}}, \boldsymbol{\psi}\right)+A_{2}\left(\boldsymbol{\eta}_{\mathbf{q}}, \mathbf{p}\right)-A\left(\boldsymbol{\eta}_{\boldsymbol{\sigma}}, \mathbf{p}\right)+A_{1}\left(\boldsymbol{\eta}_{\boldsymbol{\sigma}}, \phi\right)+J\left(\eta_{u}, \phi\right) .
$$

Using (4.1)-(4.3), we arrive at

$$
\begin{aligned}
\left\|\eta_{u}\right\|^{2}= & A\left(\boldsymbol{\eta}_{\mathbf{q}}, \boldsymbol{\theta}_{\boldsymbol{\psi}}\right)-A_{1}\left(\eta_{u}, \boldsymbol{\theta}_{\boldsymbol{\psi}}\right)+J_{1}\left(\boldsymbol{\eta}_{\boldsymbol{\sigma}}, \boldsymbol{\theta}_{\boldsymbol{\psi}}\right)+A_{2}\left(\boldsymbol{\eta}_{\mathbf{q}}, \boldsymbol{\theta}_{\mathbf{p}}\right)-A\left(\boldsymbol{\eta}_{\boldsymbol{\sigma}}, \boldsymbol{\theta}_{p}\right) \\
& +A_{1}\left(\boldsymbol{\eta}_{\boldsymbol{\sigma}}, \theta_{\phi}\right)+J\left(\eta_{u}, \theta_{\phi}\right)-\int_{0}^{t} B\left(t, s, \boldsymbol{\eta}_{\mathbf{q}}, \mathbf{I}_{h} \mathbf{p}\right) d s
\end{aligned}
$$

where $\theta_{\phi}=\phi-I_{h} \phi, \boldsymbol{\theta}_{\mathbf{p}}=\mathbf{p}-\mathbf{I}_{h} \mathbf{p}$ and $\boldsymbol{\theta}_{\boldsymbol{\psi}}=\boldsymbol{\psi}-\Pi_{h} \boldsymbol{\psi}$. Using the Cauchy-Schwarz inequality, Lemma 2.1, Lemma 2.3 and the fact that $\|\mathbf{p}\|_{\mathbf{H}^{1}(\Omega)} \leq M\|\boldsymbol{\psi}\|_{\mathbf{H}^{1}(\Omega)}$, we obtain

$$
\left|A\left(\boldsymbol{\eta}_{\mathbf{q}}, \boldsymbol{\theta}_{\boldsymbol{\psi}}\right)+A_{2}\left(\boldsymbol{\eta}_{\mathbf{q}}, \boldsymbol{\theta}_{\mathbf{p}}\right)+A\left(\boldsymbol{\eta}_{\boldsymbol{\sigma}}, \boldsymbol{\theta}_{\mathbf{p}}\right)\right| \leq C\left[\sum_{K} \frac{h_{K}^{2}}{p_{K}^{2}}\left(\left\|\boldsymbol{\eta}_{\mathbf{q}}\right\|_{K}^{2}+\left\|\boldsymbol{\eta}_{\boldsymbol{\sigma}}\right\|_{K}^{2}\right)\right]^{\frac{1}{2}}\|\boldsymbol{\psi}\|_{\mathbf{H}^{1}(\Omega)} .
$$

Proceeding similarly as for finding (4.15), it yields

$$
\left|A_{1}\left(\eta_{u}, \boldsymbol{\theta}_{\boldsymbol{\psi}}\right)\right| \leq C\left(\sum_{K} \frac{h_{K}^{2}}{p_{K}^{2}}\left\|\nabla \theta_{u}\right\|_{K}^{2}+\sum_{e_{k} \in \Gamma} C_{11}^{-1} h_{K} \int_{e_{k}} C_{11} \llbracket \eta_{u} \rrbracket^{2} d S\right)^{\frac{1}{2}}\|\boldsymbol{\psi}\|_{\mathbf{H}^{1}(\Omega)},
$$

and

$$
\begin{aligned}
\left|A_{1}\left(\boldsymbol{\eta}_{\boldsymbol{\sigma}}, \theta_{\phi}\right)\right| & =\mid \sum_{K} \int_{K} \boldsymbol{\eta}_{\boldsymbol{\sigma}} \cdot \nabla \theta_{\phi} d x-\int_{\Gamma}\left(\left\{\left\{\boldsymbol{\eta}_{\boldsymbol{\sigma}}\right\}-C_{12} \llbracket \boldsymbol{\eta}_{\boldsymbol{\sigma}} \rrbracket\right) \llbracket \theta_{\phi} \rrbracket d S \mid\right. \\
& \leq\left|\sum_{K} \int_{K} \boldsymbol{\eta}_{\boldsymbol{\sigma}} \cdot \nabla \theta_{\phi} d x\right|+\mid \int_{\Gamma}\left(\left\{\left\{\boldsymbol{\eta}_{\boldsymbol{\sigma}}\right\}-C_{12} \llbracket \boldsymbol{\eta}_{\boldsymbol{\sigma}} \rrbracket\right) \llbracket \theta_{\phi} \rrbracket d S \mid .\right.
\end{aligned}
$$

A use of Cauchy-Schwarz and Lemma 2.1 gives

$$
\left|\sum_{K} \int_{K} \boldsymbol{\eta}_{\boldsymbol{\sigma}} \cdot \nabla \theta_{\phi} d x\right| \leq C\left[\sum_{K} \frac{h_{K}^{2}}{p_{K}^{2}}\left\|\boldsymbol{\eta}_{\boldsymbol{\sigma}}\right\|_{K}^{2}\right]^{\frac{1}{2}}\|\phi\|_{H^{2}(\Omega)} .
$$


We can rewrite the second term of (4.41) as

$$
\mid \int_{\Gamma}\left(\left\{\left\{\boldsymbol{\eta}_{\boldsymbol{\sigma}}\right\}-C_{12} \llbracket \boldsymbol{\eta}_{\boldsymbol{\sigma}} \rrbracket\right) \llbracket \theta_{\phi} \rrbracket d S \mid \leq C \sum_{e_{k} \in \Gamma}\left(\int_{e_{k}}\left\{\left|\boldsymbol{\rho}_{\boldsymbol{\sigma}}\right|\right\} \llbracket \llbracket \theta_{\phi} \rrbracket d S+\int_{e_{k}}\left\{\left|\boldsymbol{\theta}_{\boldsymbol{\sigma}}\right|\right\} \llbracket \llbracket \theta_{\phi} \rrbracket d S\right) .\right.
$$

Using trace inequality (2.4) and Lemma 2.1, we obtain

$$
\mid \int_{\Gamma}\left(\left\{\left\{\boldsymbol{\eta}_{\boldsymbol{\sigma}}\right\}-C_{12} \llbracket \boldsymbol{\eta}_{\boldsymbol{\sigma}} \rrbracket\right) \llbracket \theta_{\phi} \rrbracket d S \mid \leq C \frac{h}{p^{\frac{1}{2}}}\left(\left\|\boldsymbol{\rho}_{\boldsymbol{\sigma}}\right\|+\frac{h^{\min \{r, p\}+1}}{p^{r+1}}\|\boldsymbol{\sigma}\|_{H^{r+1}(\Omega)}\right)\|\phi\|_{H^{2}(\Omega)} .\right.
$$

A use of Cauchy-Schwarz and Lemma 2.3 yields

$$
\left|J_{1}\left(\boldsymbol{\eta}_{\boldsymbol{\sigma}}, \boldsymbol{\theta}_{\boldsymbol{\psi}}\right)\right| \leq C\left[\sum_{e_{k} \in \Gamma} C_{22} h_{K} \int_{e_{k}} C_{22} \llbracket \boldsymbol{\eta}_{\boldsymbol{\sigma}} \rrbracket^{2} d S\right]^{\frac{1}{2}}\|\boldsymbol{\psi}\|_{\mathbf{H}^{1}(\Omega)},
$$

and

$$
\left|J\left(\eta_{u}, \theta \phi\right)\right| \leq C\left[\sum_{e_{k} \in \Gamma} C_{11} \frac{h_{K}^{3}}{p_{K}^{3}} \int_{e_{k}} C_{11} \llbracket \eta_{u} \rrbracket^{2} d S\right]^{\frac{1}{2}}\|\phi\|_{H^{2}(\Omega)} .
$$

Now the last term on the right hand side of (4.38)

$$
\begin{aligned}
\left|\int_{0}^{t} B\left(t, s, \boldsymbol{\eta}_{\mathbf{q}}(s), \mathbf{I}_{h} \mathbf{p}(t)\right) d s\right| & \leq C\left(\int_{0}^{t}\left\|\boldsymbol{\eta}_{\mathbf{q}}(s)\right\|_{\left(\mathbf{H}^{1}(\Omega)\right)^{*}} d s\right)\left\|\mathbf{I}_{h} \mathbf{p}\right\|_{\mathbf{H}^{1}(\Omega)} \\
& \leq C\left(\int_{0}^{t}\left\|\boldsymbol{\eta}_{\mathbf{q}}(s)\right\|_{\left(\mathbf{H}^{1}(\Omega)\right)^{*}} d s\right)\|\mathbf{p}\|_{\mathbf{H}^{1}(\Omega)} .
\end{aligned}
$$

Substitute the estimate (4.21) of $\left\|\boldsymbol{\eta}_{\mathbf{q}}\right\|_{\mathbf{H}^{1}(\Omega)^{*}}$ from Lemma 4.1 and find that

$$
\mid \int_{0}^{t} B\left(t, s,\left(\boldsymbol{\eta}_{\mathbf{q}}(s), \mathbf{I}_{h} \mathbf{p}(t)\right) d s \mid \leq C\|\mathbf{p}\|_{\mathbf{H}^{1}(\Omega)} \int_{0}^{t}\left(\left\|\eta_{u}\right\|+C \frac{h^{(P+D)}}{p^{(Q+Z)}}\|u\|_{H^{r+2}}\right) d s .\right.
$$

From the definition we note that $\|\mathbf{p}\|_{\mathbf{H}^{1}(\Omega)} \leq C\|\phi\|_{H^{2}(\Omega)}$ and $\|\boldsymbol{\psi}\|_{\mathbf{H}^{1}(\Omega)} \leq\|\phi\|_{H^{2}(\Omega)}$. Now using the regularity condition (4.34), estimates (4.39) - (4.47) and Lemma 4.1, we obtain

$$
\left\|\eta_{u}\right\| \leq C \frac{h^{P+D}}{p^{Q+Z}}\left(\|u\|_{H^{r+2}(\Omega)}+\int_{0}^{t}\|u\|_{H^{r+2}(\Omega)} d s\right)+\int_{0}^{t}\left\|\eta_{u}\right\| d s .
$$

An application of Gronwall's Lemma gives the estimate (4.32) for $\left\|u-\tilde{u}_{h}\right\|$. For the estimate (4.33), we now differentiate (4.1)-(4.3) with respect to time. Then, we proceed as above and use (4.31) to complete the rest of the proof.

\section{Error Estimates}

In this section, we prove our main theorem, that is Theorem 3.1. Using extended mixed Ritz-Volterra projection, we write

$$
\begin{gathered}
u-u_{h}=\left(u-\tilde{u}_{h}\right)-\left(u_{h}-\tilde{u}_{h}\right)=\eta_{u}-\xi_{u}, \\
\mathbf{q}-\mathbf{q}_{h}=\left(\mathbf{q}-\tilde{\mathbf{q}}_{h}\right)-\left(\mathbf{q}_{h}-\tilde{\mathbf{q}}_{h}\right)=\boldsymbol{\eta}_{\mathbf{q}}-\boldsymbol{\xi}_{\mathbf{q}} \\
\boldsymbol{\sigma}-\boldsymbol{\sigma}_{h}=(\boldsymbol{\sigma}-\tilde{\boldsymbol{\sigma}})-\left(\boldsymbol{\sigma}_{h}-\tilde{\boldsymbol{\sigma}}\right)=\boldsymbol{\eta}_{\boldsymbol{\sigma}}-\boldsymbol{\xi}_{\boldsymbol{\sigma}}
\end{gathered}
$$


Since we have already estimated $\eta_{u}, \boldsymbol{\eta}_{\mathbf{q}}$ and $\boldsymbol{\eta}_{\boldsymbol{\sigma}}$, now we only have to find the estimates for $\xi_{u}$, $\boldsymbol{\xi}_{\mathbf{q}}$ and $\boldsymbol{\xi}_{\boldsymbol{\sigma}}$. Using (4.1)-(4.3), the system (3.24)-(3.26) can be written as

$$
\begin{array}{rlrl}
A\left(\boldsymbol{\xi}_{\mathbf{q}}, \mathbf{w}_{h}\right)-A_{1}\left(\xi_{u}, \mathbf{w}_{h}\right)+J_{1}\left(\boldsymbol{\xi}_{\boldsymbol{\sigma}}, \boldsymbol{\xi}_{\boldsymbol{\sigma}}\right) & =0 & & \forall \mathbf{w}_{h} \in \mathbf{w}_{h}, \\
A_{2}\left(\boldsymbol{\xi}_{\mathbf{q}}, \boldsymbol{\tau}_{h}\right)-A\left(\boldsymbol{\xi}_{\boldsymbol{\sigma}}, \boldsymbol{\tau}_{h}\right)+\int_{0}^{t} B\left(t, s, \boldsymbol{\xi}_{\mathbf{q}}(s), \boldsymbol{\tau}_{h}\right) d s & =0 & \forall \boldsymbol{\tau}_{h} \in \mathbf{w}_{h}, \\
\left(\xi_{u_{t}}, v_{h}\right)+A_{1}\left(\boldsymbol{\xi}_{\boldsymbol{\sigma}}, v_{h}\right)+J\left(\xi_{u}, v_{h}\right) & =\left(\eta_{u_{t}}, v_{h}\right) & \forall v_{h} \in V_{h} .
\end{array}
$$

Below, we establish estimates for $\left\|\xi_{u}\right\|,\left\|\boldsymbol{\xi}_{\mathbf{q}}\right\|$ and $\left\|\boldsymbol{\xi}_{\boldsymbol{\sigma}}\right\|$.

Lemma 5.1. There exist a constant $C$ independent of $h$ and $p$ such that

$$
\begin{aligned}
\left\|\xi_{u}\right\|^{2}+\int_{0}^{t}\left\|\boldsymbol{\xi}_{\mathbf{q}}\right\|^{2} d s & \leq C\left(\left\|\xi_{u}(0)\right\|^{2}+\int_{0}^{T}\left\|\eta_{u_{t}}\right\|^{2} d s\right) \\
\left\|\boldsymbol{\xi}_{\mathbf{q}}\right\|^{2}+\left\|\boldsymbol{\xi}_{\boldsymbol{\sigma}}\right\|^{2} & \leq C\left(\left\|\boldsymbol{\xi}_{\mathbf{q}}(0)\right\|^{2}+J\left(\xi_{u}(0), \xi_{u}(0)\right)\right)+\int_{0}^{T}\left\|\eta_{u_{t}}\right\|^{2} d s .
\end{aligned}
$$

Proof. Choose $\mathbf{w}_{h}=\boldsymbol{\xi}_{\boldsymbol{\sigma}}$ in (5.1), $\boldsymbol{\tau}_{h}=\boldsymbol{\xi}_{\mathbf{q}}$ in (5.2) and $v_{h}=\xi_{u}$ in (5.3) and add to obtain

$$
\frac{1}{2} \frac{d}{d t}\left\|\xi_{u}\right\|^{2}+A_{2}\left(\boldsymbol{\xi}_{\mathbf{q}}, \boldsymbol{\xi}_{\mathbf{q}}\right)+J\left(\xi_{u}, \xi_{u}\right)+J_{1}\left(\boldsymbol{\xi}_{\boldsymbol{\sigma}}, \boldsymbol{\xi}_{\boldsymbol{\sigma}}\right)=\left(\eta_{u_{t}}, \xi_{u}\right)-\int_{0}^{t} B\left(t, s, \boldsymbol{\xi}_{\mathbf{q}}(s), \boldsymbol{\xi}_{\mathbf{q}}\right) d s
$$

Using $a \geq \alpha_{0}$ and Cauchy-Schwarz inequality, we arrive at

$$
\frac{d}{d t}\left\|\xi_{u}\right\|^{2}+\alpha_{0}\left\|\boldsymbol{\xi}_{\mathbf{q}}\right\|^{2}+2 J\left(\xi_{u}, \xi_{u}\right)+2 J_{1}\left(\boldsymbol{\xi}_{\boldsymbol{\sigma}}, \boldsymbol{\xi}_{\boldsymbol{\sigma}}\right) \leq\left\|\eta_{u_{t}}\right\|^{2}+\left\|\xi_{u}\right\|^{2}+C(T) \int_{0}^{t}\left\|\boldsymbol{\xi}_{\mathbf{q}}(s)\right\|^{2} d s
$$

Integrate from 0 to $t$ to obtain

$$
\begin{aligned}
\left\|\xi_{u}\right\|^{2}+\int_{0}^{t}\left\{\left\|\boldsymbol{\xi}_{\mathbf{q}}\right\|^{2}+J\left(\xi_{u}, \xi_{u}\right)+J_{1}\left(\boldsymbol{\xi}_{\boldsymbol{\sigma}}, \boldsymbol{\xi}_{\boldsymbol{\sigma}}\right)\right\} d s & \leq\left\|\xi_{u}(0)\right\|^{2}+C \int_{0}^{t}\left\|\eta_{u_{t}}\right\|^{2} d s \\
& +\alpha_{0} \int_{0}^{t}\left\{\left\|\xi_{u}\right\|^{2}+\int_{0}^{s}\left\|\boldsymbol{\xi}_{\mathbf{q}}(\tau)\right\|^{2} d \tau\right\} d s .
\end{aligned}
$$

A use of Gronwall's Lemma yields

$$
\left\|\xi_{u}\right\|^{2}+\int_{0}^{t}\left\|\boldsymbol{\xi}_{\mathbf{q}}\right\|^{2} d s \leq C\left(\left\|\xi_{u}(0)\right\|^{2}+\int_{0}^{T}\left\|\eta_{u_{t}}\right\|^{2} d s\right) .
$$

Now differentiate equation (5.1) with respect to t and choose $\mathbf{w}_{h}=\boldsymbol{\xi}_{\boldsymbol{\sigma}}$ in (5.1), $\boldsymbol{\tau}_{h}=\boldsymbol{\xi}_{\mathbf{q} t}$ in (5.2) and $v_{h}=\xi_{u_{t}}$ in (5.3). Then add and use Cauchy-Schwarz inequality to obtain

$$
\begin{aligned}
\frac{1}{2} \frac{d}{d t} A_{2}\left(\boldsymbol{\xi}_{\mathbf{q}}, \boldsymbol{\xi}_{\mathbf{q}}\right) & +\frac{1}{2}\left\|\xi_{u_{t}}\right\|^{2}+\frac{1}{2} \frac{d}{d t} J\left(\xi_{u}, \xi_{u}\right)+J_{1}\left(\boldsymbol{\xi}_{\boldsymbol{\sigma}}, \boldsymbol{\xi}_{\boldsymbol{\sigma}}\right) \leq \frac{1}{2}\left\|\eta_{u_{t}}\right\|^{2} \\
& -\frac{d}{d t} \int_{0}^{t} B\left(t, s, \boldsymbol{\xi}_{\mathbf{q}}(s), \boldsymbol{\xi}_{\mathbf{q}}\right) d s+B\left(t, t, \boldsymbol{\xi}_{\mathbf{q}}(t), \boldsymbol{\xi}_{\mathbf{q}}\right)+\int_{0}^{t} B_{t}\left(t, s, \boldsymbol{\xi}_{\mathbf{q}}(s), \boldsymbol{\xi}_{\mathbf{q}}\right) d s .
\end{aligned}
$$


Integrating from 0 to $t$ and using the boundedness of $B$ and the property $a \geq \alpha_{0}$, we arrive at

$$
\begin{aligned}
\left\|\boldsymbol{\xi}_{\mathbf{q}}\right\|^{2}+\int_{0}^{t}\left(\left\|\xi_{u_{t}}\right\|^{2}\right. & \left.+J_{1}\left(\boldsymbol{\xi}_{\boldsymbol{\sigma}}, \boldsymbol{\xi}_{\boldsymbol{\sigma}}\right)\right) d s+J\left(\xi_{u}, \xi_{u}\right) \leq \alpha_{0}\left\|\boldsymbol{\xi}_{\mathbf{q}}(0)\right\|^{2}+J\left(\xi_{u}(0), \xi_{u}(0)\right)+\int_{0}^{t}\left\|\eta_{u_{t}}\right\|^{2} d s \\
& -\int_{0}^{t} B\left(t, s, \boldsymbol{\xi}_{\mathbf{q}}(s), \boldsymbol{\xi}_{\mathbf{q}}(t)\right) d s+\int_{0}^{t} B\left(s, s, \boldsymbol{\xi}_{\mathbf{q}}(s), \boldsymbol{\xi}_{\mathbf{q}}(s)\right) d s \\
& +\int_{0}^{t} \int_{0}^{s} B_{s}\left(s, \tau, \boldsymbol{\xi}_{\mathbf{q}}(\tau), \boldsymbol{\xi}_{\mathbf{q}}(s)\right) d \tau d s \\
& \leq \alpha_{0}\left\|\boldsymbol{\xi}_{\mathbf{q}}(0)\right\|^{2}+J\left(\xi_{u}(0), \xi_{u}(0)\right)+C\left\|\boldsymbol{\xi}_{\mathbf{q}}(t)\right\| \int_{0}^{t}\left\|\boldsymbol{\xi}_{\mathbf{q}}(s)\right\| d s \\
& +C \int_{0}^{t}\left\|\boldsymbol{\xi}_{\mathbf{q}}(s)\right\|^{2} d s+C \int_{0}^{t}\left\{\left\|\boldsymbol{\xi}_{\mathbf{q}}(s)\right\| \int_{0}^{s}\left\|\boldsymbol{\xi}_{\mathbf{q}}(\tau)\right\| d \tau\right\} d s+\int_{0}^{t}\left\|\eta_{u_{t}}\right\|^{2} d s
\end{aligned}
$$

Using Young's inequality appropriately, we obtain

$$
\begin{aligned}
\left\|\boldsymbol{\xi}_{\mathbf{q}}\right\|^{2}+\int_{0}^{t}\left\|\xi_{u_{t}}\right\|^{2}+J\left(\xi_{u}, \xi_{u}\right) \leq & C\left(\alpha_{0}, T\right)\left(\left\|\boldsymbol{\xi}_{\mathbf{q}}(0)\right\|^{2}+J\left(\xi_{u}(0), \xi_{u}(0)\right)+\int_{0}^{T}\left\|\eta_{u_{t}}\right\|^{2} d s\right) \\
& +C\left(\alpha_{0}, T\right) \int_{0}^{t}\left\|\boldsymbol{\xi}_{\mathbf{q}}(s)\right\|^{2} d s .
\end{aligned}
$$

Again a use of Gronwall's Lemma yields

$$
\left\|\boldsymbol{\xi}_{\mathbf{q}}\right\|^{2}+J\left(\xi_{u}, \xi_{u}\right) \leq C\left(\left\|\boldsymbol{\xi}_{\mathbf{q}}(0)\right\|^{2}+J\left(\xi_{u}(0), \xi_{u}(0)\right)+\int_{0}^{t}\left\|\eta_{u_{t}}\right\|^{2} d s\right) .
$$

In (5.2) taking $\boldsymbol{\tau}_{h}=\boldsymbol{\xi}_{\boldsymbol{\sigma}}$, using Cauchy-Schwarz inequality, we obtain

$$
\left\|\boldsymbol{\xi}_{\boldsymbol{\sigma}}\right\| \leq C\left(\left\|\boldsymbol{\xi}_{\mathbf{q}}\right\|+\int_{0}^{t}\left\|\boldsymbol{\xi}_{\mathbf{q}}(s)\right\| d s\right)
$$

and using (5.7), we obtain the desired estimates. This completes the proof.

Proof of Theorem 3.1. Using the triangle inequality, we can write

$$
\left\|u-u_{h}\right\| \leq\left\|u-\tilde{u}_{h}\right\|+\left\|\tilde{u}_{h}-u_{h}\right\| .
$$

Now a use of Lemma 4.2 and Lemma 5.1 with the choices $\tilde{u}_{h}(0)=I_{h} u_{0}$ and $\tilde{\mathbf{q}}_{h}(0)=I_{h} \nabla u_{0}$ gives the estimate (3.27) of $\left\|u-u_{h}\right\|$. In the similar way, we can find the estimate (3.28) for $\left\|\mathbf{q}-\mathbf{q}_{h}\right\|$ and $\left\|\boldsymbol{\sigma}-\boldsymbol{\sigma}_{h}\right\|$. This completes the rest of the proof.

Remark 5.1. Although for simplicity of exposition, we have discussed semidiscrete method, but the analysis for completely discrete schemes using backward Euler method and Crank-Nicolson method will not pose any special problem, see Thomée [17]. Therefore, we have omitted the discussion on completely discrete schemes.

\section{$6 \quad$ Numerical Experiments}

In this section, we discuss the performance of the proposed LDGFEM methods for the following linear parabolic problem:

$$
\begin{aligned}
u_{t}-\nabla \cdot\left(\nabla u+\int_{0}^{t} e^{(t-s)} \nabla u d s\right) & =f & & \text { in } \Omega \times(0, T], \\
u & =0 & & \text { on } \partial \Omega \times(0, T], \\
u(x, 0) & =u_{0}(x) & & \text { in } \Omega,
\end{aligned}
$$


where $\Omega=(0,1) \times(0,1), T=1$ and $f$ is taken in such a way that the exact solution is $u=e^{t} x(x-1) y(y-1)$. We divide $\Omega$ into regular uniform closed triangles and $0=t_{0}<t_{1}<$ $\cdots<t_{M}=T$ be a given partition of the time interval $(0, T]$ with step length $\Delta t=\frac{T}{M}$ for some positive integer $M$. Let $U^{n}$ denote the approximation of $u_{h}$ at $t=t_{n}$. We will investigate the convergence of LDG for $\left\|u-u_{h}\right\|$ and $\left\|\mathbf{q}-\mathbf{q}_{\mathbf{h}}\right\|$. Let us first discuss the numerical procedure for the above mentioned method. Let $\left(\phi_{i}\right)_{i=1}^{N_{h}}$ be the basis functions for the finite dimensional space $V_{h}$, where $N_{h}$ denotes the dimension of the space $V_{h}$ and $\left(\chi_{i}\right)_{i=1}^{M_{h}}$ be the basis functions for the finite dimensional space $\mathbf{W}_{h}$, where $M_{h}$ denotes the dimension of the space $\mathbf{W}_{h}$. Then, we define the following matrices

$$
\begin{aligned}
M & =[M(i j)]_{1 \leq i, j \leq N_{h}}, \quad A_{1}=\left[A_{1}(i j)\right]_{1 \leq i \leq N_{h}, 1 \leq j \leq M_{h},} \\
J_{1} & =\left[J_{1}(i j)\right]_{1 \leq i, j \leq M_{h}}, \quad B=[B(i j)]_{1 \leq i, j \leq N_{h}}, \quad J=[J(i j)]_{1 \leq i, j \leq N_{h}},
\end{aligned}
$$

and the vector

$$
L=[L(i)]_{1 \leq i \leq N_{h}}
$$

where

$$
\begin{aligned}
& M(i j)=\sum_{K} \int_{K} \phi_{i} \phi_{j} d x, \quad A_{1}(i j)=\sum_{K} \int_{K} \phi_{i} \cdot \nabla \chi_{j} d x-\int_{\Gamma} \llbracket\left\{\chi_{j}\right\} \rrbracket \llbracket \phi_{i} \rrbracket d S, \\
& J_{1}(i j)=\sum_{e \in \Gamma} \int_{e} C_{22} \llbracket \chi_{i} \rrbracket \llbracket \chi_{j} \rrbracket d S, \quad B(i j)(t)=\int_{\Omega} \chi_{i} \cdot \chi_{j} d x, \quad J(i j)=\sum_{e \in \Gamma} \int_{e} C_{11} \llbracket \phi_{i} \rrbracket \llbracket \phi_{j} \rrbracket d S,
\end{aligned}
$$

and $L(i)=\int_{\Omega} f \phi_{i} d x$

Write $u_{h}=\sum_{i=1}^{N_{h}} \alpha_{i} \phi_{i}$, where $\boldsymbol{\alpha}=\left[\alpha_{1}, \alpha_{2} \cdots, \alpha_{N_{h}}\right], \mathbf{q}_{h}=\sum_{i=1}^{M_{h}} \beta_{i} \chi_{i}$, where $\boldsymbol{\beta}=\left[\beta_{1}, \beta_{2} \cdots \beta_{M_{h}}\right]$ and $\boldsymbol{\sigma}_{h}=\sum_{i=1}^{M_{h}} \gamma_{i} \boldsymbol{\chi}_{i}$, where $\boldsymbol{\gamma}=\left[\gamma_{1}, \gamma_{2} \cdots \gamma_{M_{h}}\right]$

Set

$$
\frac{\partial u_{h}^{n}}{\partial t} \approx \frac{U^{n}-U^{n-1}}{\Delta t} .
$$

Now using the basis function for $V_{h}$ and $\mathbf{W}_{h},(3.18)-(3.20)$ can be reduced to the following matrix form.

$$
\begin{aligned}
B \boldsymbol{\beta}^{n}+A_{1} \boldsymbol{\alpha}^{n}+J_{1} \boldsymbol{\gamma}^{n} & =0 \\
B \boldsymbol{\beta}^{n}-B \boldsymbol{\gamma}^{n}+\int_{0}^{t_{n}} e^{(t-s)} B \boldsymbol{\beta}^{n} d s & =0 \\
(M+d t J) \boldsymbol{\alpha}^{n}-d t A_{1}^{\prime} \boldsymbol{\gamma}^{n} & =M \alpha^{n-1}+d t L
\end{aligned}
$$

Convergence of $\left\|u-u_{h}\right\|$ and $\left\|\mathbf{q}-\mathbf{q}_{h}\right\|$. We show the orders of convergence in the $L^{2}$-norm of the error in the gradient $\mathbf{q}=\nabla u$ and in the $L^{2}$-norm of the error in $u$ in the Table 2 and Table 3 , respectively. We observe that the optimal order of convergence predicted by our theory (see Table 1) is achieved. We have plotted $-\log \left\|\mathbf{q}-\mathbf{q}_{h}\right\|$ and $-\log \left\|u-u_{h}\right\|$ with $-\log (h)$ in Figure 1 and Figure 2, respectively which confirm the theoretical result. 


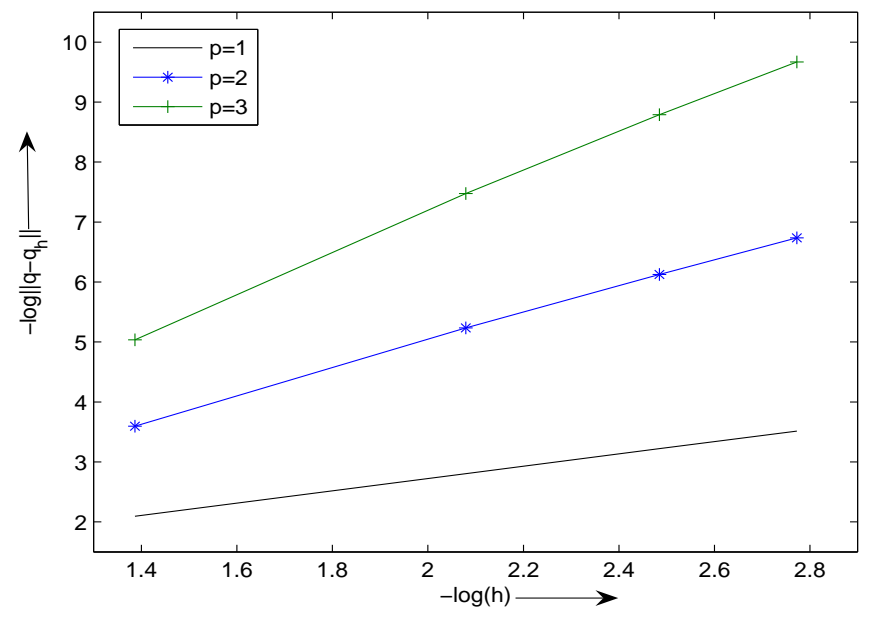

Figure 1: $L^{2}$ norm error estimates for $\mathbf{q}$ when $C_{11}=O\left(h^{-1}\right)$ and $C_{22}=O(h)$

Table 2: Table for $L^{2}$-error estimate in $\mathbf{q}$

\begin{tabular}{|c|c|c|c|c|c|c|}
\hline$C_{11} \rightarrow$ & $O(1)$ & $O(1)$ & $O(1)$ & $O\left(h^{-1}\right)$ & $O\left(h^{-1}\right)$ & $O\left(h^{-1}\right)$ \\
$C_{22} \rightarrow$ & 0 & $O(1)$ & $O(h)$ & 0 & $O(1)$ & $O(h)$ \\
\hline $\mathrm{p}=1$ & 1.1642 & 1.1751 & 1.1642 & 1.0249 & 1.0249 & 1.0250 \\
\hline $\mathrm{p}=2$ & 2.4045 & 2.4264 & 2.3352 & 2.2861 & 2.2862 & 2.2862 \\
\hline $\mathrm{p}=3$ & 3.1231 & 2.9687 & 3.5015 & 3.4137 & 3.4122 & 3.3930 \\
\hline
\end{tabular}

Table 3: Table for $L^{2}$-error estimate in $\mathrm{u}$

\begin{tabular}{|c|c|c|c|c|c|c|}
\hline$C_{11} \rightarrow$ & $O(1)$ & $O(1)$ & $O(1)$ & $O\left(h^{-1}\right)$ & $O\left(h^{-1}\right)$ & $O\left(h^{-1}\right)$ \\
$C_{22} \rightarrow$ & 0 & $O(1)$ & $O(h)$ & 0 & $O(1)$ & $O(h)$ \\
\hline $\mathrm{p}=1$ & 2.3457 & 2.3579 & 2.3157 & 2.2341 & 2.2345 & 2.2341 \\
\hline $\mathrm{p}=2$ & 3.2440 & 3.2444 & 3.2440 & 3.1635 & 3.1599 & 3.1633 \\
\hline $\mathrm{p}=3$ & 4.0343 & 3.9996 & 4.0343 & 4.0234 & 3.9940 & 4.0200 \\
\hline
\end{tabular}

\section{Conclusion}

In this paper, we have proposed and analyzed an $h p$-LDG method for a parabolic type integrodifferential equation. Compared to the elliptic case [3], [15], we have, in this article, established similar $h p$-error estimates for the semidiscrete scheme after suitably modifying the numerical flux $\hat{u}$. Due to the presence of integral term, an introduction of an expanded mixed RitzVolterra projection helps to achieve optimal estimates. Further, a dual norm estimate plays a crucial role in our error analysis. Finally, we have also discussed some numerical results. As a consequence of the present analysis, it is easy to derive error estimates for parabolic problems. 
Acknowledgment: The authors gratefully acknowledge the research support of the Department of Science and Technology, Government of India vide project No. 08DST012. They also acknowledge Professor Neela Nataraj for her valuable suggestions and help on Numerical experiments. This publication is also based on work supported in part by Award No. KUK-C1-013-04, made by King Abdullah University of Science and Technology (KAUST).

\section{References}

[1] Babuska, I. and Suri, M., The hp-version of the finite element method with quasi-uniform meshes, RAIRO Model. Math. Anal. Nume. 21, 1987, 199-238.

[2] Cannon, J. R. and Lin, Y., Nonclassical $H^{1}$ projections and Galerkin methods for nonlinear parabolic integro-differential equations, CALCOLO 25, 1988, 187-201.

[3] Castillo, P., Cockburn, B., Perugia, I. and Schötzau, D., An a priori error analysis of the local discontinuous Galerkin method for elliptic problems, SIAM J. Numer. Anal. 38, 2000, 1676-1706.

[4] Castillo, P., Cockburn, B., Schötzau, D. and Schwab, C., Optimal a priori error estimates for the hp-version of the local discontinuous Galerkin method for convection-diffusion problems, Math. Comp. 71, 2002, 455-478.

[5] Cockburn, B. and Shu, C.-W., The local discontinuous Galerkin finite element method for convection-diffusion system, SIAM J.Numer. Anal. 35, 1998, 2440-2463.

[6] Ewing, R.E., Lin, Y., Sun, T., Wang, J. and Zhang, S., Sharp $L^{2}$-error estimates and super convergence of mixed finite element methods for non-Fickian flows in porous media, SIAM J. Numer. Anal. 40, 2002, 1538-1560.

[7] Ewing, R.E., Lin, Y. and Wang, J., A numerical approximation of non-Fickian flows with mixing length growth in porous media, Acta. Math. Univ. Comenian. 70, 2001, 75-84.

[8] Gudi, T., Nataraj, N. and Pani, A. K., An hp-local discontinuous Galerkin method for some quasilinear elliptic boundary value problems of nonmonotone type, Math. Comp. 77, 2007, 731-756.

[9] Levy, D., Shu, C.-W. and Yan, J., Local discontinuous Galerkin methods for nonlinear dispersive equations, J. Comput. Phys. 196, 2004, 751-772.

[10] Lin, Y., Thomée, V. and Wahlbin, L.B., Ritz-Volterra projections to finite element spaces and applications to integro-differential and related equations, SIAM J. Numer. Anal. 28, 1991, 1040-1070.

[11] Pani, A. K. and Fairweather, G., $H^{1}$-Galerkin Mixed Finite Element Methods for Parabolic Partial Integro-Differential Equations, IMA J. Numer. Anal. 22, 2002, 231-252.

[12] Pani, A. K. and Peterson, T.E., Finite element methods with numerical quadrature for parabolic integro- differential equations, SIAM J. Numer. Anal. 33, 1996, 1084-1105.

[13] Pani, A. K. and Sinha, R. K., Error estimate for semidiscrete Galerkin approximation to a time dependent parabolic integro-differential equation with nonsmooth data, CALCOLO 37, 2000, 181-205. 


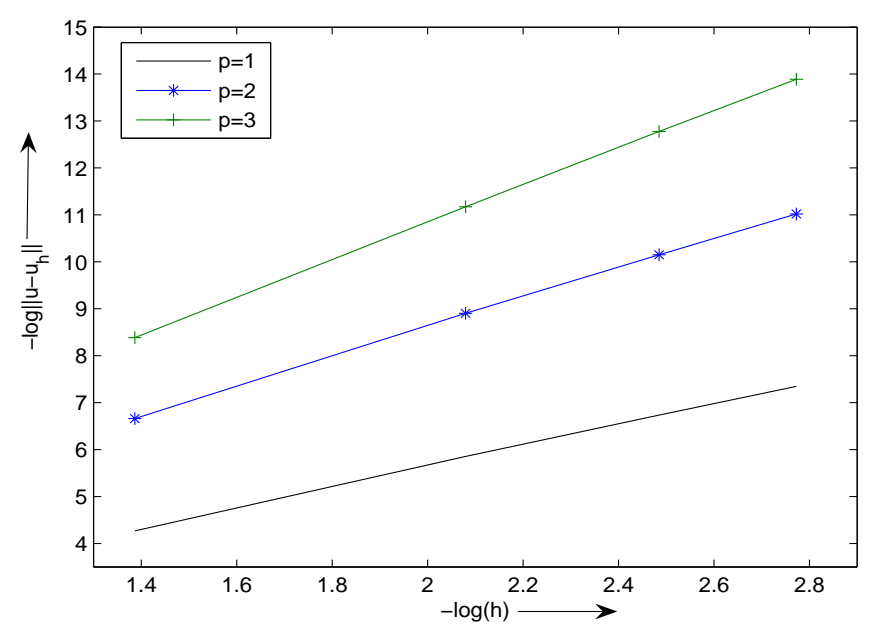

Figure 2: $L^{2}$ norm error estimates for $u$ when $C_{11}=O\left(h^{-1}\right)$ and $C_{22}=O(h)$

[14] Pani, A. K., Thomée, V. and Wahlbin L. B., Numerical methods for hyperbolic and parabolic integro-differential equations, J. Integral Equations Appl. 4 1992, 533-584.

[15] Perugia, I. and Schötzau, D., An hp-analysis of the local discontinuous Galerkin method for diffusion problems, J. Sci. Comput. 17, 2002, 561-571.

[16] Riviere, B., Wheeler, M. F. and Girault, V., A priori error estimates for finite element methods based on discontinuous approximation spaces for elliptic problems, SIAM J. Numer. Anal. 39, 2001, 902-931.

[17] Thomée, Vidar, Galerkin Finite Element methods for parabolic problems, Springer, 2006.

[18] Thomée, V. and Zhang, N. Y., Error estimates for semidiscrete finite element methods for parabolic integro-differential equations, Math. Comp. 53, 1989, 121-139.

[19] Xu, Y., Shu, C.-W., Error estimates of the semi-discrete local discontinuous Galerkin method for nonlinear convection-diffusion and KdV equations, Comput. Methods Appl. Mech. Engrg. 196, 2007, 3805-3822.

[20] Xu, Y. and Shu, C.-W., Local discontinuous Galerkin methods for high-order timedependent partial differential equations, Communications in Computational Physics 7, 2010, $1-46$.

Department of Mathematics, Industrial Mathematics Group, Indian Institute of Technology Bombay, Powai, Mumbai-400076

E-mail address: akp@math.iitb.ac.in

Department of Mathematics, Industrial Mathematics Group, Indian Institute of Technology Bombay, Powai, Mumbai-400076

E-mail address: sangita@math.iitb.ac.in 


\section{RECENT REPORTS}

06/09 Inherent noise can facilitate coherence in collective swarm motion

07/09 Solving the Coupled System Improves Computational Efficiency of the Bidomain Equations

08/09 Model reduction using a posteriori analysis

09/09 Equilibrium Order Parameters of Liquid Crystals in the LaudauDe Gennes Theory

10/09 Landau-De Gennes theory of nematic liquid crystals: the OseenFrank limit and beyond

11/09 A Comparison of Numerical Methods used for Finite Element Modelling of Soft Tissue Deformation

12/09 From Individual to Collective Behaviour of Unicellular Organisms: Recent Results and Open Problems

13/09 Stochastic modelling of reaction-diffusion processes: algorithms for bimolecular reactions

14/09 Chaste: a test-driven approach to software development for physiological modelling

15/09 Block triangular preconditioners for PDE constrained optimization

16/09 From microscopic to macroscopic descriptions of cell migration on growing domains

17/09 The Influence of Gene Expression Time Delays on GiererMeinhardt Pattern Formation Systems

18/09 Analysis of a stochastic chemical system close to a sniper bifurcation of its mean field model

19/09 On the existence and the applications of modified equations for stochastic differential equations
Yates et al.

Southern

Plank

Vigmond

Whiteley

Whiteley

Majumdar

Majumdar

Zarnescu

Pathmanathan

Gavaghan

Whiteley

Xue

Othmer

Erban

Erban

Chapman

Pitt-Francis et al.

Rees

Stoll

Baker

Yates

Erban

Seirin Lee

Gaffney

Monk

Erban et al.

Zygalakis 
20/09 Pebble bed: reflector treatment and pressure velocity coupling

21/09 A finite difference method for free boundary problems

22/09 Tangent unit-vector fields: nonabelian homotopy invariants and the Dirichlet energy

23/09 Morphological instability of a nonequilibrium icecolloid interface

24/09 The effect of polar lipids on tear film dynamics

25/09 Preconditioning for active set and projected gradient methods as semi-smooth Newton methods for PDE-constrained optimization with control constraints

26/09 Functional differential equations arising in cell-growth

27/09 A Cell Growth Model Revisited

28/09 Quasi-steady state reduction of molecular motor-based models of directed intermittent search

29/09 All-at-once preconditioning in PDE-constrained optimization
Charpin et al. Fornberg

Majumdar

Robbins

Zyskin

Peppin

Majumdar

Wettlaufer

Aydemir

Breward

Witelski

Stoll

Wathen

Wake

Begg

Derfel

van Brunt

Wake

Newby

Bressloff

Rees

Stoll

Wathen

Copies of these, and any other OCCAM reports can be obtained from:

Oxford Centre for Collaborative Applied Mathematics Mathematical Institute

24 - 29 St Giles'

Oxford

OX1 3LB

England

www.maths.ox.ac.uk/occam 